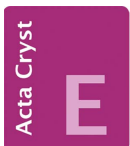

CRYSTALLOGRAPHIC COMMUNICATIONS

ISSN 2056-9890

Received 1 August 2021

Accepted 9 November 2021

Keywords: crystal structure; terbium(III); copper(II); metallamacrocycle; 15-metallacrown-5.

CCDC reference: 2121203

Supporting information: this article has supporting information at journals.iucr.org/e

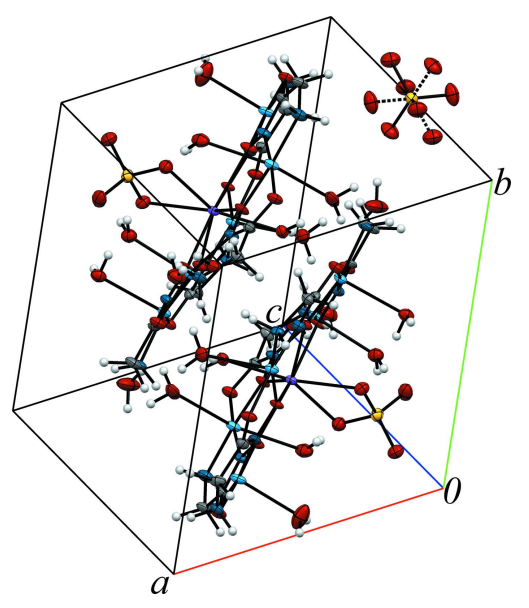

OPEN Ә ACCESS

\section{Crystal structure of a Tb"'-Cu" glycinehydroxamate 15-metallacrown-5 sulfate complex}

\author{
Anna V. Pavlishchuk, ${ }^{\text {a,b* }}$ Inna V. Vasylenko, ${ }^{\text {b }}$ Matthias Zeller ${ }^{c}$ and Anthony W. \\ Addison $^{\text {d }}$
}

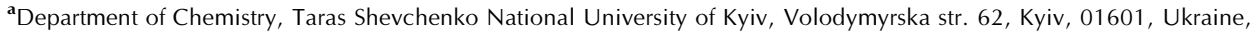
${ }^{\mathbf{b}}$ L.V. Pisarzhevskii Institute of Physical Chemistry of the National Academy of Sciences of the Ukraine, Prospect Nauki 31, Kiev 03028, Ukraine, 'Department of Chemistry, Purdue University, 560 Oval Drive, West Lafayette, IA 47907-2084, USA, and ${ }^{\mathbf{d}}$ Department of Chemistry, Drexel University, Philadelphia, PA 19104-2816, USA. *Correspondence e-mail: annpavlis@ukr.net

The core of the title complex, bis[hexaaquahemiaquapentakis $\left(\mu_{3}\right.$-glycinehydroxamato)sulfatopentacopper(II)terbium(III)] sulfate hexahydrate, $\left[\mathrm{TbCu}_{5}\left(\mathrm{SO}_{4}\right)(\mathrm{GlyHA})_{5}\left(\mathrm{H}_{2} \mathrm{O}\right)_{6.5}\right]_{2}\left(\mathrm{SO}_{4}\right) \cdot 6 \mathrm{H}_{2} \mathrm{O}$ (1), which belongs to the 15-metallacrown-5 family, consists of five glycinehydroxamate dianions GlyHA $^{2-} ; \mathrm{C}_{2} \mathrm{H}_{4} \mathrm{~N}_{2} \mathrm{O}_{2}$ ) and five copper(II) ions linked together forming a metallamacrocyclic moiety. The terbium(III) ion is connected to the centre of the metallamacrocycle through five hydroxamate oxygen atoms. The coordination environment of the $\mathrm{Tb}^{3+}$ ion is completed to an octacoordination level by oxygen atoms of a bidentate sulfate and an apically coordinated water molecule, while the copper(II) atoms are square-planar, penta- or hexacoordinate due to the apical coordination of water molecules. Continuous shape calculations indicate that the coordination polyhedron of the $\mathrm{Tb}^{3+}$ ion in $\mathbf{1}$ is best described as square antiprismatic. The positive charge of each pair of $\left[\mathrm{TbCu}_{5}\left(\mathrm{GlyHA}_{5^{-}}\right.\right.$ $\left.\left(\mathrm{H}_{2} \mathrm{O}\right)_{6.5}\left(\mathrm{SO}_{4}\right)\right]_{2}^{2+}$ fragments is compensated by a non-coordinated sulfate anion, which is located on an inversion center with 1:1 disordered oxygen atoms. Complex $\mathbf{1}$ is isomorphous with the previously reported compounds $\left[\mathrm{LnCu} \mathrm{C}^{-}\right.$ $\left.(\mathrm{GlyHA})_{5}\left(\mathrm{SO}_{4}\right)\left(\mathrm{H}_{2} \mathrm{O}\right)_{6.5}\right]_{2}\left(\mathrm{SO}_{4}\right)$, where $L n^{\mathrm{III}}=\mathrm{Pr}, \mathrm{Nd}, \mathrm{Sm}, \mathrm{Eu}, \mathrm{Gd}$, Dy and Ho.

\section{Chemical context}

Numerous research studies devoted to polynuclear $3 d-4 f$ assemblies have been stimulated by their non-trivial luminescence properties (Jankolovits et al., 2011; Maity et al., 2015), single-molecule magnet (SMM) behaviour (Dhers et al., 2016; Zangana et al., 2014) and their significant magnetocaloric effect (Pavlishchuk \& Pavlishchuk, 2020; Zheng et al., 2014). The 15-metallacrown-5 complexes are $3 d-4 f$ metallamacrocyclic assemblies, which can be easily obtained from one-step reactions between an $\alpha$-substituted hydroxamic acid and the corresponding salts of transition metals and lanthanides (Stemmler et al., 1999; Pavlishchuk et al., 2011, 2019). Compounds bearing 15-metallacrown-5 $\left\{\operatorname{LnCu}_{5}\right\}^{3+}$ units have demonstrated the ability to serve as sensors (Zabrodina et al., 2018), can absorb and adsorb various small molecules (Lim et al., 2010; Pavlishchuk et al., 2014; Ostrowska et al., 2016) and display SMM behaviour (Wang et al., 2019, 2021; Zaleski et al., 2006; Wu et al., 2021). Taking into account the fact that 15-metallacrowns-5 are also suitable building blocks for the generation of porous coordination polymers and discrete assemblies (Pavlishchuk et al., 2017a,b, 2018), the synthesis of 
new examples of this class of metallamacrocyclic assemblies and studies of their structural features are of particular interest. Herein we report the crystal structure of the new 15-metallacrown-5 complex $\left[\mathrm{TbCu}_{5}(\mathrm{GlyHA})_{5}\left(\mathrm{H}_{2} \mathrm{O}\right)_{6.5}\left(\mathrm{SO}_{4}\right)\right]_{2}$ $\left(\mathrm{SO}_{4}\right) \cdot 13\left(\mathrm{H}_{2} \mathrm{O}\right)$ (1), which complements the previously reported series of isomorphous metallamacrocycles with $\mathrm{Pr}$, Nd, Sm, Eu, Gd, Dy and Ho ions at their centres.

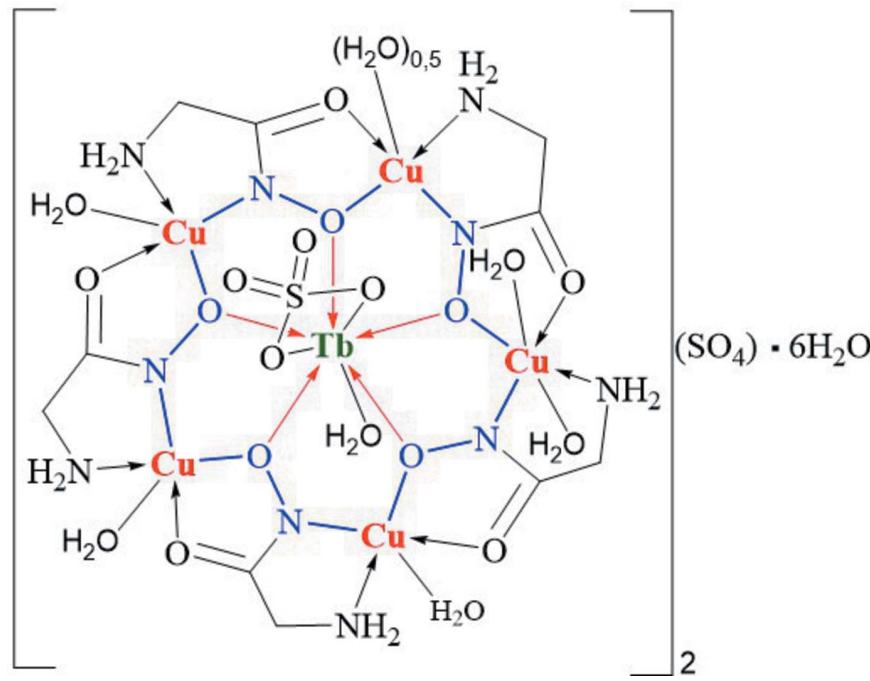

\section{Structural commentary}

Complex $\mathbf{1}$ crystallizes in the space group $P \overline{1}$ and is isostructural with the previously reported complexes

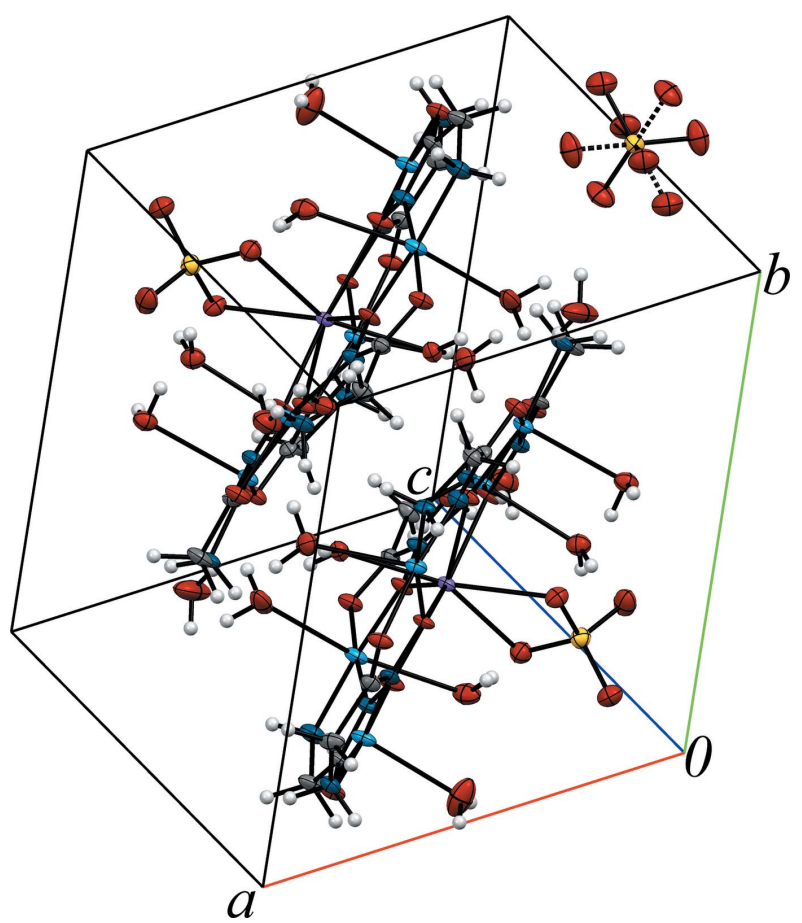

Figure 1

The unit cell of complex $\mathbf{1}$ containing two $\left[\mathrm{TbCu}_{5}(\mathrm{GlyHA})_{5^{-}}\right.$ $\left.\left(\mathrm{SO}_{4}\right)\left(\mathrm{H}_{2} \mathrm{O}\right)_{6.5}\right]^{+}$metallacrown cations and non-coordinated sulfate anions (located on a inversion center with $\mathrm{O}$ atoms 1:1 disordered). Non-coordinated water molecules are omitted for clarity of presentation.
Table 1

Selected bond lengths $(\AA)$.

\begin{tabular}{llll}
\hline $\mathrm{Cu} 1-\mathrm{N} 3$ & $1.915(4)$ & $\mathrm{Cu} 4-\mathrm{O} 8$ & $1.940(3)$ \\
$\mathrm{Cu} 1-\mathrm{O} 1$ & $1.928(3)$ & $\mathrm{Cu} 4-\mathrm{O} 7$ & $1.947(3)$ \\
$\mathrm{Cu} 1-\mathrm{O} 2$ & $1.969(3)$ & $\mathrm{Cu} 4-\mathrm{N} 10$ & $2.012(4)$ \\
$\mathrm{Cu} 1-\mathrm{N} 4$ & $1.991(4)$ & $\mathrm{Cu} 4-\mathrm{O} 17$ & $2.481(4)$ \\
$\mathrm{Cu} 1-\mathrm{O} 20$ & $2.601(4)$ & $\mathrm{Cu} 5-\mathrm{N} 1$ & $1.890(4)$ \\
$\mathrm{Cu} 1-\mathrm{O} 21$ & $2.736(4)$ & $\mathrm{Cu} 5-\mathrm{O} 9$ & $1.943(3)$ \\
$\mathrm{Cu} 2-\mathrm{N} 5$ & $1.900(4)$ & $\mathrm{Cu} 5-\mathrm{O} 10$ & $1.946(3)$ \\
$\mathrm{Cu} 2-\mathrm{O} 3$ & $1.928(3)$ & $\mathrm{Cu} 5-\mathrm{N} 2$ & $2.003(4)$ \\
$\mathrm{Cu} 2-\mathrm{O} 4$ & $1.936(3)$ & $\mathrm{Cu} 5-\mathrm{O} 18$ & $2.379(4)$ \\
$\mathrm{Cu} 2-\mathrm{N} 6$ & $2.018(4)$ & $\mathrm{Tb} 1-\mathrm{O} 9$ & $2.370(3)$ \\
$\mathrm{Cu} 2-\mathrm{O} 19$ & $2.409(10)$ & $\mathrm{Tb} 1-\mathrm{O} 1$ & $2.372(3)$ \\
$\mathrm{Cu} 3-\mathrm{N} 7$ & $1.904(4)$ & $\mathrm{Tb} 1-\mathrm{O} 15$ & $2.383(3)$ \\
$\mathrm{Cu} 3-\mathrm{O} 6$ & $1.944(3)$ & $\mathrm{Tb} 1-\mathrm{O} 3$ & $2.386(3)$ \\
$\mathrm{Cu} 3-\mathrm{O} 5$ & $1.949(3)$ & $\mathrm{Tb} 1-\mathrm{O} 7$ & $2.411(3)$ \\
$\mathrm{Cu} 3-\mathrm{N} 8$ & $2.014(4)$ & $\mathrm{Tb} 1-\mathrm{O} 5$ & $2.430(3)$ \\
$\mathrm{Cu} 3-\mathrm{O} 16$ & $2.508(4)$ & $\mathrm{Tb} 1-\mathrm{O} 12$ & $2.436(3)$ \\
$\mathrm{Cu} 4-\mathrm{N} 9$ & $1.894(4)$ & $\mathrm{Tb} 1-\mathrm{O} 11$ & $2.451(3)$ \\
\hline
\end{tabular}

$\left[\operatorname{LnCu}_{5}(\mathrm{GlyHA})_{5}\left(\mathrm{SO}_{4}\right)\left(\mathrm{H}_{2} \mathrm{O}\right)_{6.5}\right]_{2}\left(\mathrm{SO}_{4}\right)$, where $\mathrm{GlyHA}^{2-}$ is the dianion of glycinehydroxamic acid and $L n^{\mathrm{III}}=\mathrm{Pr}, \mathrm{Nd}, \mathrm{Sm}$, Eu, Gd, Dy and Ho (Pavlishchuk et al., 2011). Each unit cell in 1 contains two $\left[\mathrm{TbCu}_{5}(\mathrm{GlyHA})_{5}\left(\mathrm{SO}_{4}\right)\left(\mathrm{H}_{2} \mathrm{O}\right)_{6.5}\right]^{+}$15-metallacrown-5 cations related by an inversion center, one noncoordinated sulfate anion for charge-balance and non-coordinated water molecules (Figs. 1 and 2).

The core of the $\left[\mathrm{TbCu}_{5}(\mathrm{GlyHA})_{5}\left(\mathrm{SO}_{4}\right)\left(\mathrm{H}_{2} \mathrm{O}\right)_{6.5}\right]^{+}$complex cation in $\mathbf{1}$ is constructed from five copper(II) ions linked by five bridging glycinehydroxamate dianions $\left(\mathrm{GlyHA}^{2-}\right)$ and a terbium(III) ion bound at the centre of the metallocycle (Fig. 1). The copper(II) equatorial coordination environment in $\mathbf{1}$ is formed by two oxygen atoms (from a carboxylate and a deprotonated hydroxamate group) and two nitrogen atoms (from an amine and a deprotonated hydroxamate). The equatorial $\mathrm{Cu}-\mathrm{O}_{\mathrm{eq}}$ and $\mathrm{Cu}-\mathrm{N}_{\mathrm{eq}}$ distances range from

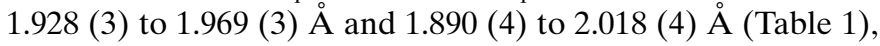
respectively, which is typical of aminohydroxamate 15-metallacrown-5 complexes (Stemmler et al., 1999; Pavlishchuk et al., 2011; Katkova et al., 2015a; Meng et al., 2016). As a result of the apical coordination of water molecules to copper(II) ions, $\mathrm{Cu} 1$ has distorted square-bipyramidal coordination [Cu1$\mathrm{O} 20=2.601$ (4) $\AA$ and $\mathrm{Cu} 1-\mathrm{O} 21=2.736$ (4) $\AA$ ], while $\mathrm{Cu} 3$, $\mathrm{Cu} 4$ and $\mathrm{Cu} 5$ are in square-pyramidal environments $[\mathrm{Cu} 3-$

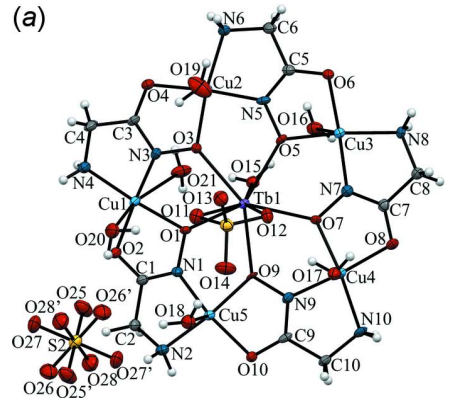

(b)

Figure 2

Structure of the $\left[\mathrm{TbCu}_{5}(\mathrm{GlyHA})_{5}\left(\mathrm{SO}_{4}\right)\left(\mathrm{H}_{2} \mathrm{O}\right)_{6.5}\right]^{+}$metallacrown cations in $\mathbf{1}$. The dashed lines indicate the disorder of the non-coordinated sulfate anion. Displacement ellipsoids are shown at the $50 \%$ probability level. [Symmetry code: (i) $x, y, z+1$.] 
Table 2

Comparison of the structural characteristics $\left(\AA{ }^{\circ}\right)$ of $\left\{L n \mathrm{Cu}_{5}\right\}^{3+} 15$-metallacrown-5 complexes with octacoordinate $L n^{\mathrm{III}}$ ions and various bidentate anions.

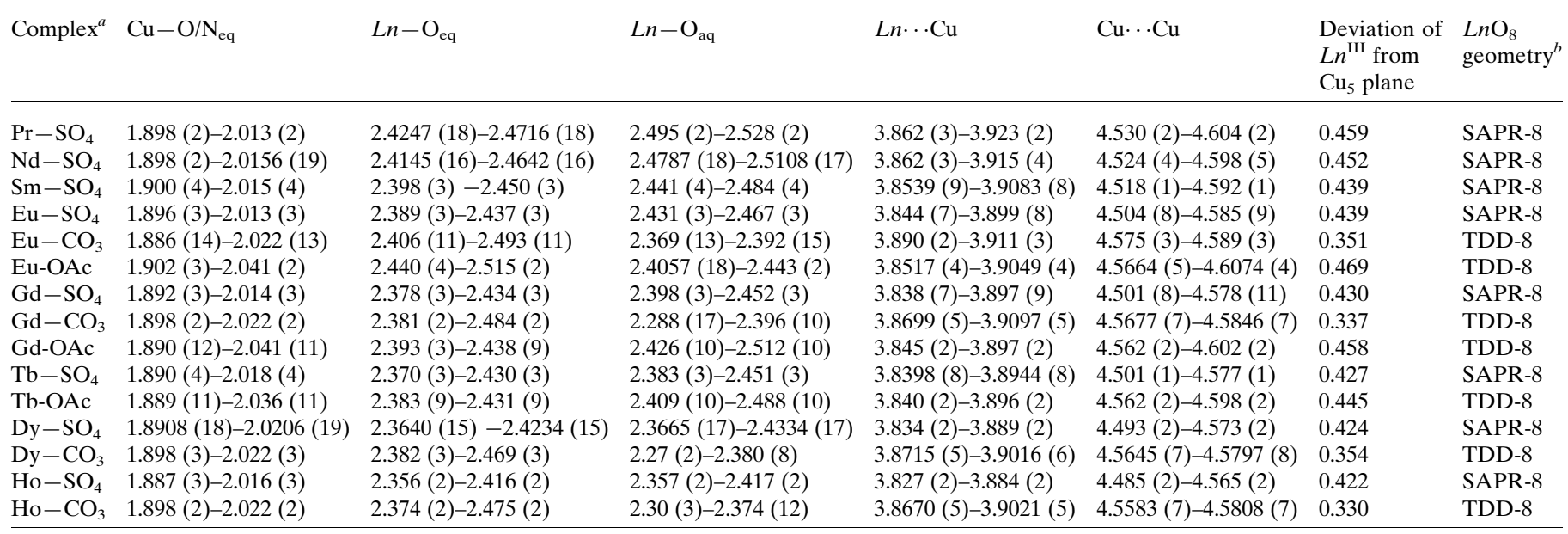

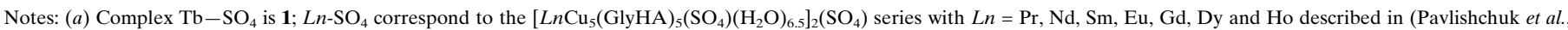

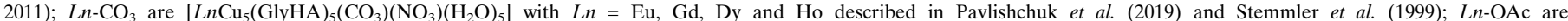

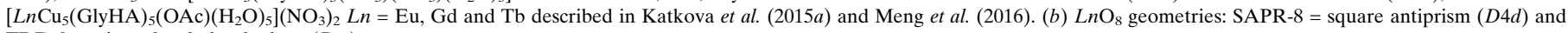
TDD-8 = triangular dodecahedron $\left(D_{2 d}\right)$.

$\mathrm{O} 16=2.508$ (4) $\AA, \mathrm{Cu} 4-\mathrm{O} 17=2.481$ (4) $\AA$ and $\mathrm{Cu} 5-\mathrm{O} 18=$ 2.379 (4) with $\tau$-values (Addison et al., 1984) ranging from 0.07 to 0.13]. As a result of the disorder of the $\mathrm{O} 19$ water molecule between two symmetry-equivalent positions with occupancy factors of $0.5,50 \%$ of the $\mathrm{Cu} 2$ atoms in $\mathbf{1}$ have square-planar coordination environments, while the other $50 \%$ possess a square-pyramidal coordination [Cu2-O19 $=2.409(10), \tau=$ 0.022 (Addison et al., 1984)]. The terbium(III) ions at the centres of the $\left[\mathrm{Cu}_{5}(\mathrm{GlyHA})_{5}\right]$ metallamacrocyclic cores in $\mathbf{1}$ are bound by five hydroxamate oxygen atoms. The $\mathrm{Tb}-\mathrm{O}_{\text {eq }}$ bond lengths are typical for 15-metallacrown-5 complexes and range from 2.370 (3) to 2.430 (3) $\AA$ (Stemmler et al., 1999; Pavlishchuk et al., 2011; Katkova et al., 2015a; Meng et al., 2016).

The coordination environment of the $\mathrm{Tb}^{3+}$ ion is completed to an octacoordination level via the two oxygen atoms O11 $[\mathrm{Tb} 1-\mathrm{O} 11=2.451(3) \AA]$ and $\mathrm{O} 12[\mathrm{~Tb} 1-\mathrm{O} 12=2.436(3) \AA]$ from the bidentate sulfate anions and $\mathrm{O} 15[\mathrm{~Tb} 1-\mathrm{O} 15=$ 2.383 (3) $\AA$ ] from a water molecule coordinated in the transposition opposite to the $\mathrm{SO}_{4}{ }^{2-}$ ion. An analysis of selected structural parameters for complex $\mathbf{1}$ and those of isomorphous compounds with other $L n^{\mathrm{III}}$ ions (Table 2) reveals the influence of the lanthanide contraction. Similar behaviour was found in other series of lanthanide(III) containing metallamacrocycles (Pavlishchuk et al., 2011; Zaleski et al., 2011). According to Shape 2.1 (Casanova et al., 2005) calculations (Fig. 3, Table 3), the coordination geometry of the $\mathrm{Tb}^{\mathrm{III}}$ ion in $\mathbf{1}$ is a square antiprism $\left(D_{4 d}\right)$, which is of particular interest with respect to potential generation of lanthanide(III)containing SMMs (Liu et al., 2018). The deviations from an idealized square-antiprismatic geometry in the $\left[\operatorname{Ln} \mathrm{Cu}_{5}(\mathrm{GlyHA})_{5}\left(\mathrm{SO}_{4}\right)\left(\mathrm{H}_{2} \mathrm{O}\right)_{6.5}\right]_{2}\left(\mathrm{SO}_{4}\right)$ complexes decrease with reduction of the deviation of the $L n^{\mathrm{III}}$ ion from the mean plane of the metallacrown core, which parallels the ionic radii of the $L n^{\mathrm{III}}$ ions (Table 3). It may be noted that, in the case of a series of related 15-metallacrown-5 complexes with octacoordinate $L n^{\text {III }}$ ions containing bidentate carbonates or acetates instead of sulfates, the coordination of the lanthanide ions is triangular dodecahedral $\left(D_{2 d}\right)$ (Table 3$)$.

The $\mathrm{Cu} \cdots \mathrm{Cu}$ and $\mathrm{Ln} \cdots \mathrm{Cu}$ separations for complex 1 range from 4.501 (1) to 4.577 (1) $\AA$ and 3.8398 (8) to 3.8944 (8) $\AA$, respectively, and are typical for $\left\{\mathrm{LnCu}_{5}\right\}^{3+}$ metallacrowns (Stemmler et al., 1999; Pavlishchuk et al., 2011; Katkova et al., 2015a; Meng et al., 2016). The $\mathrm{Cu}-\mathrm{O}, \mathrm{Cu}-\mathrm{N}$ and $\mathrm{Cu} \cdots \mathrm{Cu}$ distances do not vary significantly amongst metallamacrocycles with different bidentate counter-anions (Table 2). The metallacrown moiety in $\mathbf{1}$ is close to planar, the deviation of

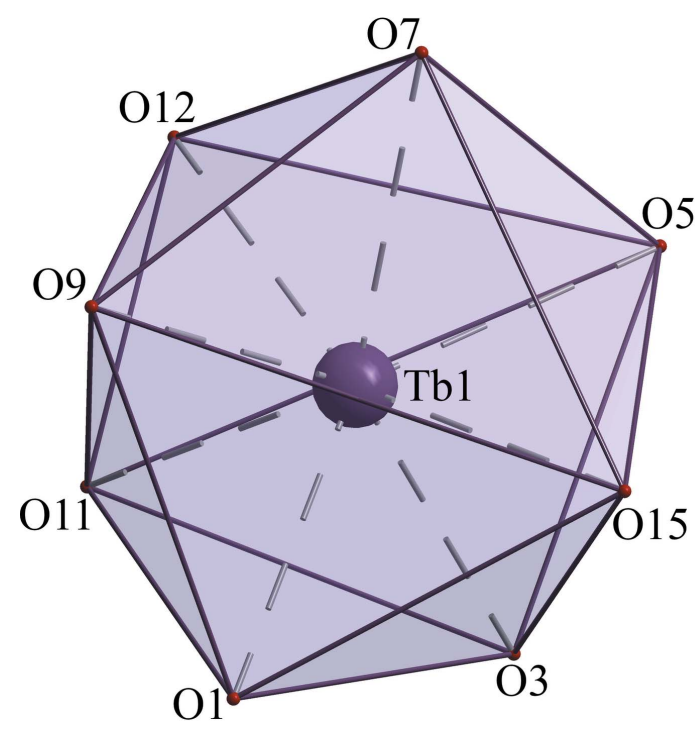

Figure 3

The $\mathrm{Tb}^{\mathrm{III}}$ coordination sphere geometry in $\mathbf{1}$. 
Table 3

Continuous shape calculations for octacoordinated $\mathrm{Ln}^{3+}$ ions in $\mathbf{1}$ obtained with Shape 2.1 software (Casanova et al., 2005).

\begin{tabular}{|c|c|c|c|c|c|c|c|c|}
\hline & OP-8 & HPY-8 & HBPY-8 & CU-8 & SAPR-8 & TDD-8 & JGBF-8 & JETBPY-8 \\
\hline $\mathrm{Pr}-\mathrm{SO}_{4}$ & 30.846 & 22.755 & 15.952 & 11.561 & 2.215 & 2.397 & 13.029 & 25.482 \\
\hline $\mathrm{Nd}-\mathrm{SO}_{4}$ & 30.677 & 22.888 & 15.968 & 11.587 & 2.141 & 2.364 & 13.033 & 25.516 \\
\hline $\mathrm{Sm}-\mathrm{SO}_{4}$ & 30.387 & 22.903 & 15.951 & 11.562 & 2.020 & 2.311 & 13.013 & 25.752 \\
\hline $\mathrm{Gd}-\mathrm{SO}_{4}$ & 30.465 & 23.110 & 16.032 & 11.570 & 1.907 & 2.269 & 13.151 & 26.121 \\
\hline $\mathrm{Tb}-\mathrm{SO}_{4}$ & 30.381 & 23.117 & 16.159 & 11.666 & 1.854 & 2.322 & 13.140 & 26.276 \\
\hline $\mathrm{Dy}-\mathrm{SO}_{4}$ & 30.357 & 23.195 & 16.112 & 11.603 & 1.799 & 2.254 & 13.168 & 26.433 \\
\hline $\mathrm{Ho}-\mathrm{SO}_{4}$ & 30.272 & 23.212 & 16.095 & 11.588 & 1.761 & 2.247 & 13.186 & 26.496 \\
\hline
\end{tabular}

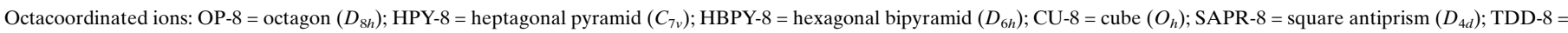
triangular dodecahedron $\left(D_{2 d}\right)$; JGBF-8 = Johnson gyrobifastigium J26 $\left(D_{2 d}\right)$; JETBPY-8 = Johnson elongated triangular bipyramid J14 $\left(D_{3 h}\right)$.

$\mathrm{Tb}^{\mathrm{III}}$ ions from the mean plane Cu1-Cu5 being $0.4270(4) \AA$. The $L n-\mathrm{O}$ distances, $L n-\mathrm{Cu}$ separations and deviations of the $L n^{\mathrm{III}}$ ions from the $\mathrm{Cu}_{5}$ planes of the metallamacrocycles trend with the lanthanide contraction in all members of the

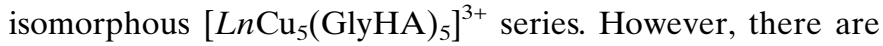
some minor differences in the observed values for a given $L n^{\text {III }}$ ion, depending on the coordinated bidentate counteranion, which is likely associated with the different planarities

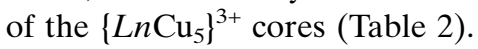

Table 4

Hydrogen-bond geometry $\left(\AA \stackrel{\circ}{\circ}^{\circ}\right)$.

\begin{tabular}{|c|c|c|c|c|}
\hline$D-\mathrm{H} \cdots A$ & $D-\mathrm{H}$ & $\mathrm{H} \cdots A$ & $D \cdots A$ & $D-\mathrm{H} \cdots A$ \\
\hline $\mathrm{O} 24-\mathrm{H} 24 B \cdots \mathrm{O} 8$ & $0.84(2)$ & $2.01(3)$ & $2.807(5)$ & $159(7)$ \\
\hline $\mathrm{O} 24-\mathrm{H} 24 A \cdots \mathrm{O} 11^{\mathrm{i}}$ & $0.84(2)$ & $2.21(3)$ & $3.015(5)$ & $162(7)$ \\
\hline $\mathrm{O} 23-\mathrm{H} 23 B \cdots \mathrm{O} 13^{\mathrm{ii}}$ & $0.85(2)$ & $2.02(3)$ & $2.853(5)$ & $166(6)$ \\
\hline $\mathrm{O} 23-\mathrm{H} 23 A \cdots \mathrm{O} 4^{\mathrm{i}}$ & $0.84(2)$ & $1.89(2)$ & $2.734(5)$ & $176(7)$ \\
\hline $\mathrm{O} 22-\mathrm{H} 22 B \cdots \mathrm{O} 23$ & $0.84(2)$ & $1.89(3)$ & $2.701(6)$ & $162(8)$ \\
\hline $\mathrm{O} 22-\mathrm{H} 22 A \cdots \mathrm{O} 26^{\mathrm{iii}}$ & $0.84(2)$ & $2.18(4)$ & $2.968(9)$ & $155(8)$ \\
\hline $\mathrm{O} 22-\mathrm{H} 22 A \cdots \mathrm{O} 28^{\mathrm{ii}}$ & $0.84(2)$ & $1.92(3)$ & $2.733(9)$ & $161(8)$ \\
\hline $\mathrm{O} 21-\mathrm{H} 21 B \cdots \mathrm{O} 10^{\mathrm{iii}}$ & $0.83(2)$ & $1.91(3)$ & $2.728(5)$ & $165(8)$ \\
\hline $\mathrm{O} 21-\mathrm{H} 21 A \cdots \mathrm{O} 18^{\mathrm{iv}}$ & $0.84(2)$ & $1.94(3)$ & $2.765(5)$ & $167(7)$ \\
\hline $\mathrm{O} 20-\mathrm{H} 20 B \cdots \mathrm{O} 11$ & $0.83(2)$ & $2.14(3)$ & $2.960(5)$ & $168(7)$ \\
\hline $\mathrm{O} 20-\mathrm{H} 20 A \cdots \mathrm{O} 26^{\mathrm{v}}$ & $0.83(2)$ & $2.09(3)$ & $2.916(9)$ & $170(7)$ \\
\hline $\mathrm{O} 20-\mathrm{H} 20 A \cdots \mathrm{O} 25$ & $0.83(2)$ & $2.02(5)$ & $2.719(9)$ & $142(7)$ \\
\hline $\mathrm{O} 19-\mathrm{H} 19 B \cdots \mathrm{O} 24^{\mathrm{vi}}$ & $0.84(2)$ & $2.07(9)$ & $2.866(11)$ & $157(22)$ \\
\hline $\mathrm{O} 19-\mathrm{H} 19 A \cdots \mathrm{O} 24^{\mathrm{vii}}$ & $0.84(2)$ & $1.72(7)$ & 2.535 (12) & $162(21)$ \\
\hline $\mathrm{O} 18-\mathrm{H} 18 B \cdots \mathrm{O} 14$ & $0.83(2)$ & $1.90(2)$ & $2.732(5)$ & $173(7)$ \\
\hline $\mathrm{O} 18-\mathrm{H} 18 A \cdots \mathrm{O} 26^{\mathrm{v}}$ & $0.84(2)$ & $2.04(3)$ & $2.857(9)$ & $163(7)$ \\
\hline $\mathrm{O} 18-\mathrm{H} 18 A \cdots \mathrm{O} 27$ & $0.84(2)$ & $1.91(4)$ & $2.648(9)$ & $146(6)$ \\
\hline $\mathrm{O} 17-\mathrm{H} 17 B \cdots \mathrm{O}^{\mathrm{vi}}$ & $0.83(2)$ & $1.90(2)$ & $2.730(5)$ & $176(7)$ \\
\hline $\mathrm{O} 17-\mathrm{H} 17 A \cdots \mathrm{O} 12$ & $0.83(2)$ & $2.10(3)$ & $2.905(5)$ & $163(6)$ \\
\hline $\mathrm{O} 16-\mathrm{H} 16 B \cdots \mathrm{O} 22$ & $0.84(2)$ & $1.89(2)$ & $2.721(6)$ & $173(7)$ \\
\hline $\mathrm{O} 16-\mathrm{H} 16 A \cdots \mathrm{O} 17^{\mathrm{iv}}$ & $0.84(2)$ & $1.95(2)$ & $2.784(5)$ & $172(7)$ \\
\hline $\mathrm{O} 15-\mathrm{H} 15 B \cdots \mathrm{O} 16$ & $0.84(2)$ & $1.86(2)$ & $2.692(5)$ & $170(6)$ \\
\hline $\mathrm{O} 15-\mathrm{H} 15 A \cdots \mathrm{O} 21$ & $0.84(2)$ & $1.85(3)$ & $2.668(5)$ & $166(6)$ \\
\hline $\mathrm{N} 10-\mathrm{H} 10 B \cdots \mathrm{O} 22^{\text {viii }}$ & 0.91 & 2.13 & $2.920(6)$ & 145 \\
\hline $\mathrm{N} 10-\mathrm{H} 10 A \cdots \mathrm{O} 20^{\mathrm{i}}$ & 0.91 & 2.24 & $2.987(5)$ & 139 \\
\hline $\mathrm{N} 8-\mathrm{H} 8 B \cdots \mathrm{O} 12^{\mathrm{vi}}$ & 0.91 & 2.04 & $2.937(5)$ & 168 \\
\hline $\mathrm{N} 8-\mathrm{H} 8 A \cdots \mathrm{O} 23$ & 0.91 & 2.20 & $3.031(5)$ & 152 \\
\hline $\mathrm{N} 6-\mathrm{H} 6 B \cdots \mathrm{O} 13^{\mathrm{ix}}$ & 0.91 & 2.64 & $3.363(5)$ & 137 \\
\hline $\mathrm{N} 6-\mathrm{H} 6 B \cdots \mathrm{O} 24^{\mathrm{vi}}$ & 0.91 & 2.24 & $2.984(6)$ & 139 \\
\hline $\mathrm{N} 6-\mathrm{H} 6 A \cdots \mathrm{O} 13^{\mathrm{iv}}$ & 0.91 & 2.25 & $3.158(5)$ & 175 \\
\hline $\mathrm{N} 4-\mathrm{H} 4 B \cdots \mathrm{O} 2^{\mathrm{x}}$ & 0.91 & 2.33 & $3.182(5)$ & 156 \\
\hline $\mathrm{N} 4-\mathrm{H} 4 A \cdots \mathrm{O} 27^{\mathrm{iv}}$ & 0.91 & 2.18 & $3.037(9)$ & 156 \\
\hline $\mathrm{N} 4-\mathrm{H} 4 A \cdots \mathrm{O} 25^{\mathrm{x}}$ & 0.91 & 2.01 & $2.789(9)$ & 143 \\
\hline $\mathrm{N} 2-\mathrm{H} 2 B \cdots \mathrm{O} 27$ & 0.91 & 2.55 & $3.418(9)$ & 159 \\
\hline $\mathrm{N} 2-\mathrm{H} 2 B \cdots \mathrm{O} 28^{\mathrm{v}}$ & 0.91 & 2.08 & $2.868(9)$ & 144 \\
\hline $\mathrm{N} 2-\mathrm{H} 2 A \cdots \mathrm{O} 15^{\mathrm{iii}}$ & 0.91 & 2.07 & $2.946(5)$ & 162 \\
\hline
\end{tabular}

Symmetry codes: (i) $x, y+1, z$; (ii) $x+1, y+1, z$; (iii) $-x+1,-y+1,-z+1$; (iv) $x+1, y, z$; (v) $-x,-y,-z+1$; (vi) $-x+1,-y+1,-z$; (vii) $x, y-1, z$; (viii) $x-1, y, z$; (ix) $-x+1,-y,-z ;(\mathrm{x})-x+1,-y,-z+1$.

\section{Supramolecular features}

The $\left[\mathrm{LnCu}_{5}(\mathrm{GlyHA})_{5}\right]^{3+}$ cations in complex $\mathbf{1}$ are non-oligomerized, which is typical for 15 -metallacrown-5 complexes. The water apical to $\mathrm{Tb}^{\mathrm{III}}$ in $\mathbf{1}(\mathrm{O} 15)$ is involved in the formation of intramolecular hydrogen bonds (O15$\mathrm{H} 15 A \cdots \mathrm{O} 21$ and $\mathrm{O} 15-\mathrm{H} 15 B \cdots \mathrm{O} 16)$ with apically coordinated water molecules $\mathrm{O} 16$ and $\mathrm{O} 21$ on copper(II) ions $\mathrm{Cu} 3$ and $\mathrm{Cu} 1$, respectively. Intramolecular hydrogen bonds in $\mathbf{1}$ are also formed between the bidentate sulfate and apically coordinated water molecules $\mathrm{O} 17, \mathrm{O} 18$ and $\mathrm{O} 20$ (O17$\mathrm{H} 17 A \cdots \mathrm{O} 12, \mathrm{O} 18-\mathrm{H} 18 B \cdots \mathrm{O} 14$ and $\mathrm{O} 20-\mathrm{H} 20 B \cdots \mathrm{O} 11)$ on copper(II) ions $\mathrm{Cu} 4, \mathrm{Cu} 5$ and $\mathrm{Cu} 1$. An extended system of intermolecular hydrogen bonds $\left[\mathrm{N} 2-\mathrm{H} 2 A \cdots \mathrm{O} 15^{\mathrm{iii}}\right.$, N8$\mathrm{H} 8 B \cdots \mathrm{O} 12^{\mathrm{vi}}\left(\mathrm{SO}_{4}\right), \mathrm{N} 10-\mathrm{H} 10 A \cdots \mathrm{O} 20^{\mathrm{i}}, \mathrm{O} 10^{\mathrm{iiii}} \cdots \mathrm{H} 21 B-$ $\mathrm{O} 21, \quad \mathrm{O}^{\mathrm{vi}} \cdots \mathrm{H} 17 B-\mathrm{O} 17, \quad \mathrm{O} 21-\mathrm{H} 21 A \cdots \mathrm{O} 18^{\mathrm{iv}}, \quad \mathrm{O} 16-$ $\left.\mathrm{H} 16 A \cdots \mathrm{O} 17^{\mathrm{iv}}\right]$ links adjacent $\left[\mathrm{TbCu}_{5}(\mathrm{GlyHA})_{5}\left(\mathrm{H}_{2} \mathrm{O}\right)_{6.5}\right.$ $\left.\left(\mathrm{SO}_{4}\right)\right]^{+}$cations and non-coordinated sulfate anions [N4$\mathrm{H} 4 A \cdots \mathrm{O} 27^{\mathrm{iv}}\left(\mathrm{SO}_{4}\right), \mathrm{O} 18-\mathrm{H} 18 A \cdots \mathrm{O} 27\left(\mathrm{SO}_{4}\right), \quad \mathrm{N} 4-\mathrm{H} 4 A \cdots$ $\mathrm{O} 25^{\mathrm{x}}\left(\mathrm{SO}_{4}\right)$ and $\left.\mathrm{O} 20-\mathrm{H} 20 A \cdots \mathrm{O} 25\left(\mathrm{SO}_{4}\right)\right]$. Non-coordinated water molecules in $\mathbf{1}$ are linked by hydrogen bonds with carbonyl oxygen and amine nitrogen atoms in the glycinehydroxamate unit from the metallacrown core $\left(\mathrm{O} 4^{i} \cdots \mathrm{H} 23 A-\right.$ $\mathrm{O} 23, \mathrm{O} 8 \cdots \mathrm{H} 24 B-\mathrm{O} 24, \mathrm{~N} 6-\mathrm{H} 6 B \cdots \mathrm{O} 24^{\mathrm{vi}}$, $\mathrm{N} 8-\mathrm{H} 8 A \cdots \mathrm{O} 23$, $\mathrm{N} 10-\mathrm{H} 10 B \cdots \mathrm{O} 22^{\text {viii }}$, apically coordinated water molecules $\left(\mathrm{O} 16-\mathrm{H} 16 B \cdots \mathrm{O} 22, \quad \mathrm{O} 19-\mathrm{H} 19 A \cdots \mathrm{O} 24^{v i i}\right.$, O19--H19B $\cdots$ $\left.\mathrm{O} 24^{v i}\right)$ or bidentate sulfate $\left(\mathrm{O} 11^{\mathrm{i}} \cdots \mathrm{H} 24 A-\mathrm{O} 24\right.$ and $\left.\mathrm{O} 13^{\mathrm{ii}} \cdots \mathrm{H} 23 B-\mathrm{O} 23\right)$. Hydrogen-bond parameters and symmetry codes are given in Table 4 .

\section{Database survey}

Compounds most closely related to $\mathbf{1}$ are its isomorphous counterparts $\left[\operatorname{LnCu}_{5}(\mathrm{GlyHA})_{5}\left(\mathrm{SO}_{4}\right)\left(\mathrm{H}_{2} \mathrm{O}\right)_{6.5}\right]_{2}\left(\mathrm{SO}_{4}\right)$, where $\mathrm{GlyHA}^{2-}$ is the dianion of glycinehydroxamic acid and $L n^{\mathrm{III}}=$ Pr, Nd, Sm, Eu, Gd, Dy and Ho (Pavlishchuk et al., 2011). A search of the Cambridge Structural Database (Version 5.41, 2021; Groom et al., 2016) reveals other compounds that also

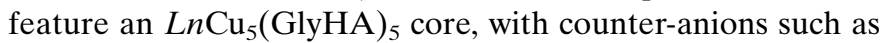
nitrate, acetate, chloride, lactate, carbonate, sulfate, isophthalate, terephthalate and all lanthanide ions other than radioactive Pm (Katkova et al., 2015a,b; Pavlishchuk et al., 2011, 2017a, Pavlishchuk et al., 2018, 2019; Stemmler et al., 1999; Muravyeva et al., 2016; Kremlev et al., 2016). Most of 
Table 5

Experimental details.

\begin{tabular}{|c|c|}
\hline \multicolumn{2}{|l|}{ Crystal data } \\
\hline Chemical formula & $\begin{array}{l}{\left[\mathrm{TbCu}_{5}\left(\mathrm{C}_{2} \mathrm{H}_{4} \mathrm{~N}_{2} \mathrm{O}_{2}\right)_{5}\left(\mathrm{SO}_{4}\right)-\right.} \\
\left.\left(\mathrm{H}_{2} \mathrm{O}\right)_{6.5}\right]_{2}\left(\mathrm{SO}_{4}\right) \cdot 6 \mathrm{H}_{2} \mathrm{O}\end{array}$ \\
\hline$M_{\mathrm{r}}$ & 2464.44 \\
\hline Crystal system, space group & Triclinic, $P \overline{1}$ \\
\hline Temperature $(\mathrm{K})$ & 150 \\
\hline$a, b, c(\AA)$ & $9.6370(4), 11.5888(5), 16.2367(6)$ \\
\hline$\alpha, \beta, \gamma\left({ }^{\circ}\right)$ & $\begin{array}{l}99.6716(13), 91.3031(12), \\
105.3123(12)\end{array}$ \\
\hline$V\left(\AA^{3}\right)$ & $1719.80(12)$ \\
\hline$Z$ & 1 \\
\hline Radiation type & $\mathrm{Cu} K \alpha$ \\
\hline$\mu\left(\mathrm{mm}^{-1}\right)$ & 15.11 \\
\hline Crystal size $(\mathrm{mm})$ & $0.20 \times 0.20 \times 0.08$ \\
\hline \multicolumn{2}{|l|}{ Data collection } \\
\hline Diffractometer & $\begin{array}{l}\text { Bruker AXS D8 Quest CMOS } \\
\text { diffractometer with PhotonII } \\
\text { charge-integrating pixel array } \\
\text { detector (CPAD) }\end{array}$ \\
\hline Absorption correction & $\begin{array}{l}\text { Multi-scan (SADABS; Krause et } \\
\quad \text { al., } 2015\end{array}$ \\
\hline$T_{\min }, T_{\max }$ & $0.454,0.754$ \\
\hline $\begin{array}{l}\text { No. of measured, independent and } \\
\text { observed }[I>2 \sigma(I)] \text { reflections }\end{array}$ & $16278,7029,6786$ \\
\hline$R_{\text {int }}$ & 0.050 \\
\hline$(\sin \theta / \lambda)_{\max }\left(\AA^{-1}\right)$ & 0.639 \\
\hline \multicolumn{2}{|l|}{ Refinement } \\
\hline$R\left[F^{2}>2 \sigma\left(F^{2}\right)\right], w R\left(F^{2}\right), S$ & $0.041,0.118,1.10$ \\
\hline No. of reflections & 7029 \\
\hline No. of parameters & 562 \\
\hline No. of restraints & 22 \\
\hline $\mathrm{H}$-atom treatment & $\begin{array}{l}\mathrm{H} \text { atoms treated by a mixture of } \\
\text { independent and constrained } \\
\text { refinement }\end{array}$ \\
\hline$\Delta \rho_{\max }, \Delta \rho_{\min }\left(\mathrm{e} \AA^{-3}\right)$ & $1.59,-1.34$ \\
\hline
\end{tabular}

Computer programs: APEX3 and SAINT (Bruker, 2018), SHELXS97 (Sheldrick, 2008), SHELXL2018/3 (Sheldrick, 2015), shelXle (Hübschle et al., 2011) and publCIF (Westrip, 2010).

these complexes feature, similar to $\mathbf{1}$, individual molecular complex cations (Katkova et al., 2015a,b; Pavlishchuk et al., 2011, 2017a, 2018, 2019; Stemmler et al., 1999; Muravyeva et al., 2016; Kremlev et al., 2016), but a small number of oligomerized examples have also been reported (Pavlishchuk et al., 2017a, 2018).

\section{Synthesis and crystallization}

Complex 1 was synthesized and crystallized according a general procedure described previously (Pavlishchuk et al., 2011). Single crystals were obtained by slow evaporation from an aqueous solution of $\mathbf{1}$.

\section{Refinement}

Crystal data, data collection and structure refinement details are summarized in Table 5. The structure is isomorphous with its Dy, Eu, Gd, Ho, Nd, Pr analogues (Pavlishchuk et al., 2011) and was solved by isomorphous replacement. The O19 water molecule is disordered over two mutually exclusive positions across an inversion center and was refined as half occupied.
The non-coordinated sulfate ion is located on an inversion center and the oxygen atoms are disordered over two sets of positions with half occupancy.

$\mathrm{C}-\mathrm{H}$ bond distances were constrained to 0.99 for aliphatic $\mathrm{CH}_{2}$ moieties. $\mathrm{N}-\mathrm{H}$ bond distances were constrained to $0.91 \AA$ for pyramidal ( $s p^{3}$-hybridized) ammonium $\mathrm{NH}_{2}^{+}$ groups. Water $\mathrm{H}$-atom positions were refined, and $\mathrm{O}-\mathrm{H}$ distances were restrained to 0.84 (2) $\AA$. The $\mathrm{H} \cdot \cdots \mathrm{H}$ distances within the $\mathrm{O} 23$ and $\mathrm{O} 24$ water molecules were further restrained to $1.35(2) \AA . U_{\text {iso }}(\mathrm{H})$ values were set to $k U_{\text {eq }}(\mathrm{C} / \mathrm{N} /$ O) with $k=1.5$ for $\mathrm{OH}$, and 1.2 for $\mathrm{CH}_{2}$ and $\mathrm{NH}_{2}^{+}$units, respectively.

\section{Acknowledgements}

This work was supported partly by the Ministry of Education and Science of Ukraine: Grant of the Ministry of Education and Science of Ukraine for perspective development of a scientific direction 'Mathematical sciences and natural sciences' at Taras Shevchenko National University of Kyiv. This material is based upon work supported by the National Science Foundation through the Major Research Instrumentation Program under Grant No. CHE 1625543 (funding for the single-crystal X-ray diffractometer). AWA thanks Drexel University for support.

\section{Funding information}

Funding for this research was provided by: National Science Foundation, Division of Materials Research (grant No. CHE 1625543 to M. Zeller); National Research Foundation of Ukraine (grant No. 2020.02/0202 to A. V. Pavlishchuk).

\section{References}

Addison, A. W., Rao, T. N., Reedijk, J., van Rijn, J. \& Verschoor, G. C. (1984). J. Chem. Soc. Dalton Trans. pp. 1349-1356.

Bruker (2018). APEX3 and SAINT. Bruker AXS Inc., Madison, Wisconsin, USA.

Casanova, D., Llunell, M., Alemany, P. \& Alvarez, S. (2005). Chem. Eur. J. 11, 1479-1494.

Dhers, S., Feltham, H. L. C., Rouzières, M., Clérac, R. \& Brooker, S. (2016). Dalton Trans. 45, 18089-18093.

Groom, C. R., Bruno, I. J., Lightfoot, M. P. \& Ward, S. C. (2016). Acta Cryst. B72, 171-179.

Hübschle, C. B., Sheldrick, G. M. \& Dittrich, B. (2011). J. Appl. Cryst. 44, 1281-1284.

Jankolovits, J., Andolina, C. M., Kampf, J. W., Raymond, K. N. \& Pecoraro, V. L. (2011). Angew. Chem. 123, 9834-9838.

Katkova, M. A., Zabrodina, G. S., Muravyeva, M. S., Khrapichev, A. A., Samsonov, M. A., Fukin, G. K. \& Ketkov, S. Yu. (2015a). Inorg. Chem. Commun. 52, 31-33.

Katkova, M. A., Zabrodina, G. S., Muravyeva, M. S., Shavyrin, A. S., Baranov, E. V., Khrapichev, A. A. \& Ketkov, S. Y. (2015b). Eur. J. Inorg. Chem. pp. 5202-5208.

Krause, L., Herbst-Irmer, R., Sheldrick, G. M. \& Stalke, D. (2015). J. Appl. Cryst. 48, 3-10.

Kremlev, K. V., Samsonov, M. A., Zabrodina, G. S., Arapova, A. V., Yunin, P. A., Tatarsky, D. A., Plyusnin, P. E., Katkova, M. A. \& Ketkov, S. Y. (2016). Polyhedron, 114, 96-100.

Lim, C., Jankolovits, J., Kampf, J. \& Pecoraro, V. (2010). Chem. Asian J. 5, 46-49. 
Liu, J.-L., Chen, Y.-C. \& Tong, M.-L. (2018). Chem. Soc. Rev. 47, 2431-2453.

Maity, M., Majee, M. C., Kundu, S., Samanta, S. K., Sañudo, E. C., Ghosh, S. \& Chaudhury, M. (2015). Inorg. Chem. 54, 9715-9726.

Meng, Y., Yang, H., Li, D., Zeng, S., Chen, G., Li, S. \& Dou, J. (2016). RSC Adv. 6, 47196-47202.

Muravyeva, M. S., Zabrodina, G. S., Samsonov, M. A., Kluev, E. A., Khrapichev, A. A., Katkova, M. A. \& Mukhina, I. V. (2016). Polyhedron, 114, 165-171.

Ostrowska, M., Fritsky, I. O., Gumienna-Kontecka, E. \& Pavlishchuk, A. V. (2016). Coord. Chem. Rev. 327-328, 304-332.

Pavlishchuk, A., Naumova, D., Zeller, M., Calderon Cazorla, S. \& Addison, A. W. (2019). Acta Cryst. E75, 1215-1223.

Pavlishchuk, A. V., Kolotilov, S. V., Fritsky, I. O., Zeller, M., Addison, A. W. \& Hunter, A. D. (2011). Acta Cryst. C67, m255-m265.

Pavlishchuk, A. V., Kolotilov, S. V., Zeller, M., Lofland, S. E. \& Addison, A. W. (2018). Eur. J. Inorg. Chem. pp. 3504-3511.

Pavlishchuk, A. V., Kolotilov, S. V., Zeller, M., Lofland, S. E., Kiskin, M. A., Efimov, N. N., Ugolkova, E. A., Minin, V. V., Novotortsev, V. M. \& Addison, A. W. (2017b). Eur. J. Inorg. Chem. pp. 48664878.

Pavlishchuk, A. V., Kolotilov, S. V., Zeller, M., Lofland, S. E., Thompson, L. K., Addison, A. W. \& Hunter, A. D. (2017a). Inorg. Chem. 56, 13152-13165.

Pavlishchuk, A. V., Kolotilov, S. V., Zeller, M., Thompson, L. K. \& Addison, A. W. (2014). Inorg. Chem. 53, 1320-1330.

Pavlishchuk, A. V. \& Pavlishchuk, V. V. (2020). Theor. Exp. Chem. 56, $1-25$.
Sheldrick, G. M. (2008). Acta Cryst. A64, 112-122.

Sheldrick, G. M. (2015). Acta Cryst. C71, 3-8.

Stemmler, A. J., Kampf, J. W., Kirk, M. L., Atasi, B. H. \& Pecoraro, V. L. (1999). Inorg. Chem. 38, 2807-2817.

Wang, J., Li, Q.-W., Wu, S.-G., Chen, Y.-C., Wan, R.-C., Huang, G.-Z., Liu, Y., Liu, J.-L., Reta, D., Giansiracusa, M. J., Wang, Z.-X., Chilton, N. F. \& Tong, M.-L. (2021). Angew. Chem. Int. Ed. 60, 5299-5306.

Wang, J., Ruan, Z.-Y., Li, Q.-W., Chen, Y.-C., Huang, G.-Z., Liu, J.-L., Reta, D., Chilton, N. F., Wang, Z.-X. \& Tong, M.-L. (2019). Dalton Trans. 48, 1686-1692.

Westrip, S. P. (2010). J. Appl. Cryst. 43, 920-925.

Wu, S.-G., Ruan, Z.-Y., Huang, G.-Z., Zheng, J.-Y., Vieru, V., Taran, G., Wang, J., Chen, Y.-C., Liu, J.-L., Ho, L. T. A., Chibotaru, L. F., Wernsdorfer, W., Chen, X.-M. \& Tong, M.-L. (2021). Chem, 7, $982-$ 992.

Zabrodina, G. S., Katkova, M. A., Samsonov, M. A. \& Ketkov, S. Y. (2018). Z. Anorg. Allg. Chem. 644, 907-911.

Zaleski, C. M., Depperman, E. C., Kampf, J. W., Kirk, M. L. \& Pecoraro, V. L. (2006). Inorg. Chem. 45, 10022-10024.

Zaleski, C. M., Lim, C.-S., Cutland-Van Noord, A. D., Kampf, J. W. \& Pecoraro, V. L. (2011). Inorg. Chem. 50, 7707-7717.

Zangana, K. H., Pineda, E. M., Vitorica-Yrezabal, I. J., McInnes, E. J. L. \& Winpenny, R. E. P. (2014). Dalton Trans. 43, 13242-13249.

Zheng, Y.-Z., Zhou, G.-J., Zheng Z., \& Winpenny, R. E. P. (2014). Chem. Soc. Rev. 43, 1462-1475. 


\section{supporting information}

Acta Cryst. (2021). E77, 1197-1202 [https://doi.org/10.1107/S2056989021011907]

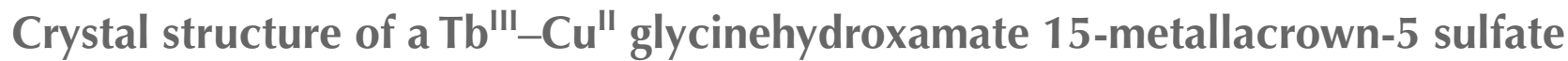 complex}

Anna V. Pavlishchuk, Inna V. Vasylenko, Matthias Zeller and Anthony W. Addison

Computing details

Data collection: APEX3 (Bruker, 2018); cell refinement: SAINT (Bruker, 2018); data reduction: SAINT (Bruker, 2018); program(s) used to solve structure: SHELXS97 (Sheldrick, 2008); program(s) used to refine structure: SHELXL2018/3 (Sheldrick, 2015), shelXle (Hübschle et al., 2011); software used to prepare material for publication: publCIF (Westrip, 2010).

Bis[hexaaquahemiaquapentakis( $\mu_{3}$-glycinehydroxamato)sulfatopentacopper(II)terbium(III)] sulfate hexahydrate

\section{Crystal data}

$\left[\mathrm{TbCu}_{5}\left(\mathrm{C}_{2} \mathrm{H}_{4} \mathrm{~N}_{2} \mathrm{O}_{2}\right)_{5}\left(\mathrm{SO}_{4}\right)\left(\mathrm{H}_{2} \mathrm{O}\right)_{6.5}\right]_{2}\left(\mathrm{SO}_{4}\right) \cdot 6 \mathrm{H}_{2} \mathrm{O}$

$M_{r}=2464.44$

Triclinic, $P \overline{1}$

$a=9.6370(4) \AA$

$b=11.5888(5) \AA$

$c=16.2367(6) \AA$

$\alpha=99.6716(13)^{\circ}$

$\beta=91.3031(12)^{\circ}$

$\gamma=105.3123(12)^{\circ}$

$V=1719.80(12) \AA^{3}$

\section{Data collection}

Bruker AXS D8 Quest CMOS diffractometer with PhotonII charge-integrating pixel array detector (CPAD)

Radiation source: I-mu-S microsource X-ray tube

Laterally graded multilayer (Goebel) mirror monochromator

Detector resolution: 7.4074 pixels $\mathrm{mm}^{-1}$

$\omega$ and phi scans
$Z=1$

$F(000)=1214$

$D_{\mathrm{x}}=2.380 \mathrm{Mg} \mathrm{m}^{-3}$

$\mathrm{Cu} K \alpha$ radiation, $\lambda=1.54178 \AA$

Cell parameters from 9965 reflections

$\theta=4.0-79.9^{\circ}$

$\mu=15.11 \mathrm{~mm}^{-1}$

$T=150 \mathrm{~K}$

Plate, blue

$0.20 \times 0.20 \times 0.08 \mathrm{~mm}$

Absorption correction: multi-scan

(SADABS; Krause et al., 2015

$T_{\min }=0.454, T_{\max }=0.754$

16278 measured reflections

7029 independent reflections

6786 reflections with $I>2 \sigma(I)$

$R_{\text {int }}=0.050$

$\theta_{\text {max }}=80.3^{\circ}, \theta_{\text {min }}=2.8^{\circ}$

$h=-12 \rightarrow 12$

$k=-14 \rightarrow 14$

$l=-19 \rightarrow 15$

562 parameters

22 restraints

Primary atom site location: structure-invariant direct methods

Secondary atom site location: difference Fourier map 
Hydrogen site location: mixed

$\mathrm{H}$ atoms treated by a mixture of independent and constrained refinement

$$
\begin{aligned}
& w=1 /\left[\sigma^{2}\left(F_{\mathrm{o}}^{2}\right)+(0.0656 P)^{2}+1.8351 P\right] \\
& \quad \text { where } P=\left(F_{\mathrm{o}}^{2}+2 F_{\mathrm{c}}^{2}\right) / 3 \\
& (\Delta / \sigma)_{\max }<0.001 \\
& \Delta \rho_{\max }=1.59 \mathrm{e} \AA^{-3} \\
& \Delta \rho_{\min }=-1.34 \mathrm{e}^{-3}
\end{aligned}
$$

Special details

Geometry. All esds (except the esd in the dihedral angle between two 1.s. planes) are estimated using the full covariance matrix. The cell esds are taken into account individually in the estimation of esds in distances, angles and torsion angles; correlations between esds in cell parameters are only used when they are defined by crystal symmetry. An approximate (isotropic) treatment of cell esds is used for estimating esds involving l.s. planes.

Refinement. The structure is ismorphous with its Dy, Eu, Gd, Ho, Nd, Pr analogues (AVP85_10mz121, AVP355_10mz172, AVP621_09mz411 and AVP629_10mz194, AVP65_10mz125 and AVP65̄1_10mz191, AVP70_10 mz147, AVP75 $10 \mathrm{mz} 148$ and AVP754_10 $\mathrm{mz} 650$ ), and was solved by isomorphous replacement. The water molecule of $\mathrm{O} 1 \overline{9}$ is disordered over two mutually exclusive positions across an inversion center and was refined as half occupied. The non-coordinated sulfate ion is located on an inversion center and the oxygen atoms are

\begin{tabular}{|c|c|c|c|c|c|}
\hline & $x$ & $y$ & $z$ & $U_{\text {iso }} * / U_{\text {eq }}$ & Occ. $(<1)$ \\
\hline $\mathrm{C} 1$ & $0.4433(5)$ & $0.2625(4)$ & $0.5042(3)$ & $0.0167(8)$ & \\
\hline $\mathrm{C} 2$ & $0.3610(5)$ & $0.2938(4)$ & 0.5788 & $0.0195(9)$ & \\
\hline $\mathrm{H} 2 \mathrm{C}$ & 0.320220 & 0.220082 & 0.602893 & $0.023^{*}$ & \\
\hline $\mathrm{H} 2 \mathrm{D}$ & 0.427084 & 0.354919 & 0.622292 & $0.023 *$ & \\
\hline $\mathrm{C} 3$ & $0.6783(5)$ & $0.0423(4)$ & 0.2493 & $0.0158(8)$ & \\
\hline $\mathrm{C} 4$ & $0.6931(6)$ & $-0.0360(5)$ & 0.3110 & $0.0235(10)$ & \\
\hline $\mathrm{H} 4 \mathrm{C}$ & 0.794720 & -0.038433 & 0.316648 & $0.028 *$ & \\
\hline H4D & 0.632740 & -0.119964 & 0.290399 & $0.028 *$ & \\
\hline $\mathrm{C} 5$ & $0.7890(5)$ & $0.3483(4)$ & 0.0317 & $0.0175(9)$ & \\
\hline C6 & $0.8858(5)$ & $0.2707(4)$ & -0.0019 & $0.0221(10)$ & \\
\hline $\mathrm{H} 6 \mathrm{C}$ & 0.874472 & 0.253424 & -0.063895 & $0.027^{*}$ & \\
\hline H6D & 0.987712 & 0.315424 & 0.015213 & $0.027^{*}$ & \\
\hline $\mathrm{C} 7$ & $0.5254(5)$ & 0.6985 (4) & 0.1304 (3) & $0.0159(8)$ & \\
\hline $\mathrm{C} 8$ & $0.6022(5)$ & $0.7507(4)$ & $0.0594(3)$ & $0.0179(9)$ & \\
\hline $\mathrm{H} 8 \mathrm{C}$ & 0.532673 & 0.736705 & 0.010561 & $0.022 *$ & \\
\hline H8D & 0.643978 & 0.839491 & 0.076935 & $0.022 *$ & \\
\hline C9 & $0.2149(5)$ & 0.5808 & 0.3895 & $0.0163(9)$ & \\
\hline $\mathrm{C} 10$ & $0.1644(5)$ & 0.6898 (4) & $0.3802(3)$ & $0.0222(10)$ & \\
\hline $\mathrm{H} 10 \mathrm{C}$ & 0.058903 & 0.664428 & 0.366493 & $0.027 *$ & \\
\hline H10D & 0.184336 & 0.748022 & 0.434014 & $0.027 *$ & \\
\hline $\mathrm{Cu} 1$ & $0.57451(7)$ & $0.15475(6)$ & $0.38923(4)$ & $0.01792(16)$ & \\
\hline $\mathrm{Cu} 2$ & $0.71639(7)$ & $0.16393(6)$ & $0.12404(4)$ & $0.01884(16)$ & \\
\hline $\mathrm{Cu} 3$ & $0.68477(7)$ & $0.53540(6)$ & $0.08031(4)$ & $0.01553(15)$ & \\
\hline $\mathrm{Cu} 4$ & $0.34975(7)$ & $0.64627(6)$ & $0.24858(4)$ & $0.01548(15)$ & \\
\hline $\mathrm{Cu} 5$ & $0.28203(7)$ & $0.39948(6)$ & $0.44370(4)$ & $0.01615(15)$ & \\
\hline $\mathrm{Tb} 1$ & $0.48345(2)$ & $0.35681(2)$ & $0.24306(2)$ & $0.01322(9)$ & \\
\hline N1 & $0.4245(4)$ & $0.3141(3)$ & $0.4416(2)$ & $0.0171(7)$ & \\
\hline
\end{tabular}
disordered over two sets of positions with half occupancy.

Water $\mathrm{H}$ atom positions were refined and $\mathrm{O}-\mathrm{H}$ distances were restrained to 0.84 (2) Angstrom, respectively. Some $\mathrm{H} \cdots \mathrm{H}$ distances were further restrained to 1.35 (2) Angstrom.

Fractional atomic coordinates and isotropic or equivalent isotropic displacement parameters $\left(\AA^{2}\right)$ 


\begin{tabular}{|c|c|c|c|c|c|}
\hline $\mathrm{N} 2$ & $0.2431(4)$ & $0.3430(4)$ & $0.5530(2)$ & $0.0195(8)$ & \\
\hline $\mathrm{H} 2 \mathrm{~A}$ & 0.236548 & 0.406266 & 0.592889 & $0.023^{*}$ & \\
\hline $\mathrm{H} 2 \mathrm{~B}$ & 0.157731 & 0.284301 & 0.547628 & $0.023^{*}$ & \\
\hline N3 & $0.6105(4)$ & $0.1242(3)$ & $0.2732(2)$ & $0.0178(7)$ & \\
\hline N4 & $0.6482(5)$ & $0.0108(4)$ & $0.3943(3)$ & $0.0215(8)$ & \\
\hline $\mathrm{H} 4 \mathrm{~A}$ & 0.724750 & 0.031948 & 0.432837 & $0.026^{*}$ & \\
\hline $\mathrm{H} 4 \mathrm{~B}$ & 0.577960 & -0.048780 & 0.410697 & $0.026^{*}$ & \\
\hline N5 & $0.7027(4)$ & $0.3075(3)$ & $0.0865(2)$ & $0.0166(7)$ & \\
\hline N6 & $0.8492(4)$ & $0.1541(3)$ & $0.0304(2)$ & $0.0171(7)$ & \\
\hline H6A & 0.931299 & 0.139490 & 0.049586 & $0.021^{*}$ & \\
\hline H6B & 0.805401 & 0.091646 & -0.011692 & $0.021^{*}$ & \\
\hline N7 & $0.5575(4)$ & $0.6039(3)$ & $0.1480(2)$ & $0.0172(7)$ & \\
\hline N8 & $0.7190(4)$ & $0.6918(3)$ & $0.0356(2)$ & $0.0159(7)$ & \\
\hline $\mathrm{H} 8 \mathrm{~A}$ & 0.805912 & 0.742721 & 0.057077 & $0.019^{*}$ & \\
\hline $\mathrm{H} 8 \mathrm{~B}$ & 0.720395 & 0.676114 & -0.021109 & $0.019^{*}$ & \\
\hline N9 & $0.2929(4)$ & $0.5471(3)$ & $0.3302(2)$ & $0.0173(7)$ & \\
\hline N10 & $0.2378(4)$ & $0.7509(4)$ & $0.3133(3)$ & $0.0226(8)$ & \\
\hline $\mathrm{H} 10 \mathrm{~A}$ & 0.298699 & 0.824200 & 0.336523 & $0.027^{*}$ & \\
\hline $\mathrm{H} 10 \mathrm{~B}$ & 0.171092 & 0.763954 & 0.278010 & $0.027^{*}$ & \\
\hline $\mathrm{O} 1$ & $0.5000(3)$ & $0.2882(3)$ & $0.37163(19)$ & $0.0166(6)$ & \\
\hline $\mathrm{O} 2$ & $0.5244(4)$ & $0.1896(3)$ & $0.5060(2)$ & $0.0196(6)$ & \\
\hline $\mathrm{O} 3$ & $0.6029(4)$ & $0.1998(3)$ & $0.2163(2)$ & $0.0199(7)$ & \\
\hline $\mathrm{O} 4$ & $0.7336(4)$ & $0.0300(3)$ & $0.1769(2)$ & $0.0196(7)$ & \\
\hline O5 & $0.6158(3)$ & $0.3809(3)$ & $0.11866(19)$ & $0.0157(6)$ & \\
\hline O6 & $0.7979(4)$ & $0.4510(3)$ & $0.0074(2)$ & $0.0189(6)$ & \\
\hline O7 & $0.4861(3)$ & $0.5537(3)$ & $0.2123(2)$ & $0.0166(6)$ & \\
\hline O8 & $0.4330(3)$ & 0.7478 & 0.1689 (2) & $0.0193(6)$ & \\
\hline O9 & $0.3463(3)$ & 0.4493 & $0.3393(2)$ & $0.0170(6)$ & \\
\hline $\mathrm{O} 10$ & $0.1827(4)$ & 0.5265 & $0.4519(2)$ & $0.0195(7)$ & \\
\hline $\mathrm{O} 11$ & $0.2853(4)$ & $0.1696(3)$ & $0.2229(2)$ & $0.0238(7)$ & \\
\hline $\mathrm{O} 12$ & $0.2734(4)$ & $0.3216(3)$ & $0.1464(2)$ & $0.0205(7)$ & \\
\hline $\mathrm{O} 13$ & 0.1448 (4) & $0.1123(3)$ & $0.0876(2)$ & $0.0253(7)$ & \\
\hline $\mathrm{O} 14$ & 0.0575 (4) & $0.2189(4)$ & $0.2058(2)$ & $0.0311(8)$ & \\
\hline O15 & $0.7222(3)$ & 0.4609 & $0.3006(2)$ & $0.0184(6)$ & \\
\hline H15A & $0.767(6)$ & $0.430(5)$ & $0.331(3)$ & $0.028^{*}$ & \\
\hline H15B & $0.782(5)$ & $0.495(5)$ & $0.269(3)$ & $0.028^{*}$ & \\
\hline O16 & $0.8851(4)$ & 0.5789 & $0.1920(2)$ & $0.0254(7)$ & \\
\hline H16A & $0.960(5)$ & $0.557(6)$ & $0.184(4)$ & $0.038^{*}$ & \\
\hline H16B & $0.917(7)$ & $0.653(2)$ & $0.213(4)$ & $0.038^{*}$ & \\
\hline O17 & $0.1408(4)$ & $0.5205(3)$ & $0.1525(2)$ & $0.0220(7)$ & \\
\hline H17A & $0.163(7)$ & $0.457(4)$ & $0.156(4)$ & $0.033^{*}$ & \\
\hline H17B & $0.155(7)$ & $0.528(6)$ & $0.1031(18)$ & $0.033^{*}$ & \\
\hline $\mathrm{O} 18$ & $0.0716(4)$ & $0.2521(3)$ & $0.3767(2)$ & $0.0241(7)$ & \\
\hline H18A & $0.043(7)$ & $0.185(3)$ & $0.393(4)$ & $0.036^{*}$ & \\
\hline H18B & $0.070(7)$ & $0.236(6)$ & $0.3246(13)$ & $0.036^{*}$ & \\
\hline O19 & $0.5200(11)$ & $0.0381(11)$ & $0.0275(6)$ & $0.047(2)$ & 0.5 \\
\hline H19A & $0.472(19)$ & $-0.003(15)$ & $0.060(9)$ & $0.071 *$ & 0.5 \\
\hline H19B & $0.55(2)$ & $0.02(2)$ & $-0.021(6)$ & $0.071^{*}$ & 0.5 \\
\hline
\end{tabular}




$\begin{array}{lllll}\text { O20 } & 0.3102(4) & 0.0221(3) & 0.3519(3) & 0.0283(8) \\ \text { H20A } & 0.236(5) & 0.022(7) & 0.377(4) & 0.043^{*} \\ \text { H20B } & 0.291(8) & 0.063(6) & 0.318(4) & 0.043^{*} \\ \text { O21 } & 0.8274(4) & 0.3337(4) & 0.3966(2) & 0.0308(8) \\ \text { H21A } & 0.909(4) & 0.320(7) & 0.392(5) & 0.046^{*} \\ \text { H21B } & 0.841(8) & 0.380(6) & 0.443(3) & 0.046^{*} \\ \text { O22 } & 0.9749(5) & 0.8150(4) & 0.2711(3) & 0.0361(9) \\ \text { H22A } & 0.972(9) & 0.855(7) & 0.319(2) & 0.054^{*} \\ \text { H22B } & 0.980(9) & 0.857(6) & 0.234(4) & 0.054^{*} \\ \text { O23 } & 0.9394(4) & 0.9116(3) & 0.1345(2) & 0.0243(7) \\ \text { H23A } & 0.876(4) & 0.947(5) & 0.150(4) & 0.036^{*} \\ \text { H23B } & 1.011(4) & 0.968(4) & 0.126(4) & 0.036^{*} \\ \text { O24 } & 0.3431(7) & 0.9430(4) & 0.1264(3) & 0.0523(14) \\ \text { H24A } & 0.328(11) & 0.998(6) & 0.163(4) & 0.079^{*} \\ \text { H24B } & 0.368(10) & 0.893(6) & 0.152(4) & 0.079^{*} \\ \text { O25 } & 0.1618(8) & 0.0365(9) & 0.4920(5) & 0.0357(19) \\ \text { O26 } & -0.0300(9) & -0.0158(7) & 0.5820(5) & 0.0339(17) \\ \text { O27 } & -0.0484(9) & 0.1032(7) & 0.4781(5) & 0.0333(17) \\ \text { O28 } & -0.0592(9) & -0.1078(7) & 0.4360(5) & 0.0346(17) \\ \text { S1 } & 0.18461(12) & 0.20240(10) & 0.16476(7) & 0.0195(2) \\ \text { S2 } & 0.000000 & 0.000000 & 0.500000 & 0.0199(3) \\ \end{array}$

Atomic displacement parameters $\left(\AA^{2}\right)$

\begin{tabular}{lllllll}
\hline & $U^{11}$ & $U^{22}$ & $U^{33}$ & $U^{12}$ & $U^{13}$ & $U^{23}$ \\
\hline $\mathrm{C} 1$ & $0.0163(19)$ & $0.0162(19)$ & $0.019(2)$ & $0.0070(16)$ & $0.0042(17)$ & $0.0016(17)$ \\
$\mathrm{C} 2$ & $0.024(2)$ & $0.025(2)$ & $0.016(2)$ & $0.0143(18)$ & $0.0049(17)$ & $0.0080(18)$ \\
$\mathrm{C} 3$ & $0.0152(19)$ & $0.0150(19)$ & $0.019(2)$ & $0.0092(16)$ & $0.0030(16)$ & $0.0011(17)$ \\
$\mathrm{C} 4$ & $0.034(3)$ & $0.024(2)$ & $0.019(2)$ & $0.020(2)$ & $0.0063(19)$ & $0.0025(19)$ \\
$\mathrm{C} 5$ & $0.018(2)$ & $0.019(2)$ & $0.016(2)$ & $0.0074(17)$ & $0.0045(17)$ & $0.0023(17)$ \\
$\mathrm{C} 6$ & $0.025(2)$ & $0.018(2)$ & $0.025(2)$ & $0.0068(18)$ & $0.0113(19)$ & $0.0018(18)$ \\
$\mathrm{C} 7$ & $0.0150(19)$ & $0.0126(19)$ & $0.019(2)$ & $0.0023(15)$ & $0.0009(16)$ & $0.0027(17)$ \\
$\mathrm{C} 8$ & $0.018(2)$ & $0.017(2)$ & $0.021(2)$ & $0.0078(16)$ & $0.0038(17)$ & $0.0055(17)$ \\
$\mathrm{C} 9$ & $0.018(2)$ & $0.0165(19)$ & $0.017(2)$ & $0.0114(16)$ & $0.0011(16)$ & $-0.0027(17)$ \\
$\mathrm{C} 10$ & $0.029(2)$ & $0.024(2)$ & $0.022(2)$ & $0.0194(19)$ & $0.0081(19)$ & $0.0066(19)$ \\
$\mathrm{Cu} 1$ & $0.0252(3)$ & $0.0179(3)$ & $0.0165(3)$ & $0.0146(3)$ & $0.0054(3)$ & $0.0048(3)$ \\
$\mathrm{Cu} 2$ & $0.0252(3)$ & $0.0152(3)$ & $0.0212(4)$ & $0.0122(3)$ & $0.0111(3)$ & $0.0051(3)$ \\
$\mathrm{Cu} 3$ & $0.0188(3)$ & $0.0136(3)$ & $0.0167(3)$ & $0.0074(2)$ & $0.0067(2)$ & $0.0039(2)$ \\
$\mathrm{Cu} 4$ & $0.0173(3)$ & $0.0138(3)$ & $0.0189(3)$ & $0.0090(2)$ & $0.0052(2)$ & $0.0045(2)$ \\
$\mathrm{Cu} 5$ & $0.0188(3)$ & $0.0174(3)$ & $0.0169(3)$ & $0.0111(3)$ & $0.0069(2)$ & $0.0053(3)$ \\
$\mathrm{Tb} 1$ & $0.01436(14)$ & $0.01192(14)$ & $0.01535(15)$ & $0.00683(10)$ & $0.00396(10)$ & $0.00235(10)$ \\
$\mathrm{N} 1$ & $0.0233(18)$ & $0.0181(17)$ & $0.0138(18)$ & $0.0112(15)$ & $0.0065(14)$ & $0.0044(14)$ \\
$\mathrm{N} 2$ & $0.0220(19)$ & $0.0194(18)$ & $0.020(2)$ & $0.0106(15)$ & $0.0073(15)$ & $0.0036(15)$ \\
$\mathrm{N} 3$ & $0.0234(19)$ & $0.0150(17)$ & $0.0201(19)$ & $0.0111(15)$ & $0.0070(15)$ & $0.0071(15)$ \\
$\mathrm{N} 4$ & $0.029(2)$ & $0.0223(19)$ & $0.020(2)$ & $0.0157(16)$ & $0.0087(16)$ & $0.0072(16)$ \\
$\mathrm{N} 5$ & $0.0159(17)$ & $0.0179(17)$ & $0.0169(18)$ & $0.0087(14)$ & $0.0056(14)$ & $-0.0016(15)$ \\
$\mathrm{N} 6$ & $0.0171(17)$ & $0.0183(18)$ & $0.0193(19)$ & $0.0092(14)$ & $0.0060(14)$ & $0.0053(15)$ \\
$\mathrm{N} 7$ & $0.0192(18)$ & $0.0170(17)$ & $0.0174(18)$ & $0.0075(14)$ & $0.0036(15)$ & $0.0043(15)$ \\
& & & & & &
\end{tabular}




\begin{tabular}{|c|c|c|c|c|c|c|}
\hline N8 & $0.0212(18)$ & $0.0104(15)$ & $0.0183(18)$ & $0.0060(14)$ & $0.0061(14)$ & $0.0057(14)$ \\
\hline N9 & $0.0202(18)$ & $0.0166(17)$ & $0.0185(19)$ & $0.0108(14)$ & $0.0029(15)$ & $0.0032(15)$ \\
\hline N10 & $0.0212(19)$ & $0.0169(18)$ & $0.034(2)$ & $0.0125(15)$ & $0.0102(17)$ & $0.0039(17)$ \\
\hline $\mathrm{O} 1$ & $0.0207(15)$ & $0.0205(15)$ & $0.0148(15)$ & $0.0141(12)$ & $0.0090(12)$ & $0.0057(12)$ \\
\hline $\mathrm{O} 2$ & 0.0277 (17) & $0.0195(15)$ & $0.0172(16)$ & $0.0146(13)$ & $0.0065(13)$ & $0.0050(13)$ \\
\hline $\mathrm{O} 3$ & 0.0307 (17) & $0.0187(15)$ & $0.0182(16)$ & $0.0155(13)$ & $0.0121(13)$ & $0.0095(13)$ \\
\hline $\mathrm{O} 4$ & $0.0251(16)$ & $0.0183(15)$ & $0.0197(16)$ & $0.0123(13)$ & $0.0084(13)$ & $0.0041(13)$ \\
\hline O5 & $0.0193(14)$ & $0.0119(13)$ & $0.0197(16)$ & $0.0103(11)$ & $0.0064(12)$ & $0.0031(12)$ \\
\hline O6 & $0.0271(17)$ & $0.0166(14)$ & $0.0172(16)$ & $0.0107(13)$ & $0.0104(13)$ & $0.0053(12)$ \\
\hline $\mathrm{O} 7$ & $0.0180(14)$ & $0.0154(14)$ & $0.0205(16)$ & $0.0086(12)$ & $0.0114(12)$ & $0.0067(12)$ \\
\hline O8 & $0.0201(15)$ & $0.0193(15)$ & $0.0238(17)$ & $0.0120(12)$ & $0.0072(13)$ & $0.0070(13)$ \\
\hline O9 & $0.0204(15)$ & $0.0178(15)$ & $0.0210(16)$ & $0.0168(12)$ & $0.0109(12)$ & $0.0065(13)$ \\
\hline $\mathrm{O} 10$ & $0.0280(17)$ & $0.0209(15)$ & $0.0172(16)$ & $0.0160(13)$ & $0.0094(13)$ & $0.0081(13)$ \\
\hline O11 & $0.0247(17)$ & $0.0219(16)$ & $0.0246(18)$ & $0.0061(13)$ & $-0.0008(14)$ & $0.0042(14)$ \\
\hline $\mathrm{O} 12$ & $0.0253(16)$ & $0.0174(15)$ & $0.0180(16)$ & $0.0028(13)$ & $0.0002(13)$ & $0.0058(13)$ \\
\hline $\mathrm{O} 13$ & $0.0280(17)$ & $0.0206(16)$ & $0.0241(18)$ & $0.0028(14)$ & $0.0013(14)$ & $0.0010(14)$ \\
\hline O14 & $0.0210(17)$ & $0.043(2)$ & $0.0267(19)$ & $0.0086(16)$ & $0.0047(14)$ & $-0.0019(16)$ \\
\hline O15 & $0.0178(15)$ & $0.0222(16)$ & $0.0167(16)$ & $0.0076(12)$ & $0.0016(12)$ & $0.0041(13)$ \\
\hline O16 & $0.0190(16)$ & $0.0268(17)$ & 0.0287 (19) & $0.0047(14)$ & $0.0044(14)$ & $0.0029(15)$ \\
\hline O17 & $0.0281(17)$ & $0.0224(16)$ & $0.0183(16)$ & $0.0111(14)$ & $0.0040(14)$ & $0.0043(14)$ \\
\hline O18 & $0.0261(17)$ & $0.0227(17)$ & $0.0236(18)$ & $0.0066(14)$ & $0.0045(14)$ & $0.0040(14)$ \\
\hline O19 & $0.038(5)$ & $0.064(6)$ & $0.026(4)$ & $-0.007(4)$ & $-0.001(4)$ & $0.005(4)$ \\
\hline $\mathrm{O} 20$ & 0.0309 (19) & $0.0200(17)$ & $0.035(2)$ & $0.0066(15)$ & $0.0100(16)$ & $0.0063(15)$ \\
\hline $\mathrm{O} 21$ & 0.0287 (19) & $0.044(2)$ & $0.0241(19)$ & $0.0232(17)$ & $0.0003(15)$ & $-0.0036(16)$ \\
\hline $\mathrm{O} 22$ & $0.047(2)$ & $0.036(2)$ & $0.032(2)$ & $0.0236(19)$ & $-0.0020(19)$ & $0.0038(17)$ \\
\hline $\mathrm{O} 23$ & $0.0215(16)$ & $0.0173(15)$ & $0.035(2)$ & $0.0072(13)$ & $0.0068(15)$ & $0.0026(14)$ \\
\hline $\mathrm{O} 24$ & $0.090(4)$ & $0.039(2)$ & $0.037(2)$ & $0.043(3)$ & $-0.015(2)$ & $-0.008(2)$ \\
\hline $\mathrm{O} 25$ & $0.023(4)$ & $0.058(5)$ & $0.034(4)$ & $0.014(4)$ & $0.007(3)$ & $0.022(4)$ \\
\hline $\mathrm{O} 26$ & $0.042(4)$ & $0.034(4)$ & $0.025(4)$ & $0.011(3)$ & 0.007 (3) & $0.002(3)$ \\
\hline $\mathrm{O} 27$ & $0.038(4)$ & $0.030(4)$ & $0.041(5)$ & $0.018(3)$ & $0.007(3)$ & 0.018 \\
\hline $\mathrm{O} 28$ & $0.044(5)$ & $0.026(4)$ & $0.033(4)$ & $0.009(3)$ & $0.004(3)$ & $0.003(3)$ \\
\hline S1 & $0.0186(5)$ & $0.0195(5)$ & $0.0200(5)$ & $0.0044(4)$ & $0.0019(4)$ & $0.0037(4)$ \\
\hline S2 & $0.0189(7)$ & $0.0180(7)$ & $0.0235(8)$ & $0.0071(6)$ & $-0.0005(6)$ & $0.0026(6)$ \\
\hline
\end{tabular}

Geometric parameters $\left(\AA,{ }^{\circ}\right)$

\begin{tabular}{llll}
\hline $\mathrm{C} 1-\mathrm{N} 1$ & $1.294(6)$ & $\mathrm{Cu} 5-\mathrm{N} 2$ & $2.003(4)$ \\
$\mathrm{C} 1-\mathrm{O} 2$ & $1.298(5)$ & $\mathrm{Cu} 5-\mathrm{O} 18$ & $2.379(4)$ \\
$\mathrm{C} 1-\mathrm{C} 2$ & $1.509(6)$ & $\mathrm{Tb} 1-\mathrm{O} 9$ & $2.370(3)$ \\
$\mathrm{C} 2-\mathrm{N} 2$ & $1.480(6)$ & $\mathrm{Tb} 1-\mathrm{O} 1$ & $2.372(3)$ \\
$\mathrm{C} 2-\mathrm{H} 2 \mathrm{C}$ & 0.9900 & $\mathrm{~Tb} 1-\mathrm{O} 15$ & $2.383(3)$ \\
$\mathrm{C} 2-\mathrm{H} 2 \mathrm{D}$ & 0.9900 & $\mathrm{~Tb} 1-\mathrm{O} 3$ & $2.386(3)$ \\
$\mathrm{C} 3-\mathrm{N} 3$ & $1.301(5)$ & $\mathrm{Tb} 1-\mathrm{O} 7$ & $2.411(3)$ \\
$\mathrm{C} 3-\mathrm{O} 4$ & $1.304(6)$ & $\mathrm{Tb} 1-\mathrm{O} 5$ & $2.430(3)$ \\
$\mathrm{C} 3-\mathrm{C} 4$ & $1.488(7)$ & $\mathrm{Tb} 1-\mathrm{O} 12$ & $2.436(3)$ \\
$\mathrm{C} 4-\mathrm{N} 4$ & $1.488(6)$ & $\mathrm{Tb} 1-\mathrm{O} 11$ & $2.451(3)$ \\
$\mathrm{C} 4-\mathrm{H} 4 \mathrm{C}$ & 0.9900 & $\mathrm{~Tb} 1-\mathrm{S} 1$ & $3.0756(11)$ \\
$\mathrm{C} 4-\mathrm{H} 4 \mathrm{D}$ & 0.9900 & $\mathrm{~N} 1-\mathrm{O} 1$ & $1.396(5)$
\end{tabular}




\begin{tabular}{|c|c|c|c|}
\hline $\mathrm{C} 5-\mathrm{N} 5$ & $1.295(6)$ & $\mathrm{N} 2-\mathrm{H} 2 \mathrm{~A}$ & 0.9100 \\
\hline $\mathrm{C} 5-\mathrm{O} 6$ & $1.298(6)$ & $\mathrm{N} 2-\mathrm{H} 2 \mathrm{~B}$ & 0.9100 \\
\hline $\mathrm{C} 5-\mathrm{C} 6$ & $1.509(6)$ & $\mathrm{N} 3-\mathrm{O} 3$ & $1.389(5)$ \\
\hline C6-N6 & $1.491(6)$ & $\mathrm{N} 4-\mathrm{H} 4 \mathrm{~A}$ & 0.9100 \\
\hline $\mathrm{C} 6-\mathrm{H} 6 \mathrm{C}$ & 0.9900 & $\mathrm{~N} 4-\mathrm{H} 4 \mathrm{~B}$ & 0.9100 \\
\hline C6-H6D & 0.9900 & N5-O5 & $1.395(4)$ \\
\hline $\mathrm{C} 7-\mathrm{N} 7$ & $1.288(6)$ & N6-H6A & 0.9100 \\
\hline $\mathrm{C} 7-\mathrm{O} 8$ & $1.298(5)$ & N6-H6B & 0.9100 \\
\hline $\mathrm{C} 7-\mathrm{C} 8$ & $1.509(6)$ & $\mathrm{N} 7-\mathrm{O} 7$ & $1.388(5)$ \\
\hline $\mathrm{C} 8-\mathrm{N} 8$ & $1.489(5)$ & $\mathrm{N} 8-\mathrm{H} 8 \mathrm{~A}$ & 0.9100 \\
\hline $\mathrm{C} 8-\mathrm{H} 8 \mathrm{C}$ & 0.9900 & $\mathrm{~N} 8-\mathrm{H} 8 \mathrm{~B}$ & 0.9100 \\
\hline $\mathrm{C} 8-\mathrm{H} 8 \mathrm{D}$ & 0.9900 & N9-O9 & $1.391(5)$ \\
\hline $\mathrm{C} 9-\mathrm{O} 10$ & $1.282(6)$ & $\mathrm{N} 10-\mathrm{H} 10 \mathrm{~A}$ & 0.9100 \\
\hline C9-N9 & $1.306(6)$ & N10-H10B & 0.9100 \\
\hline $\mathrm{C} 9-\mathrm{C} 10$ & $1.498(6)$ & $\mathrm{O} 11-\mathrm{S} 1$ & $1.500(4)$ \\
\hline $\mathrm{C} 10-\mathrm{N} 10$ & $1.487(7)$ & $\mathrm{O} 12-\mathrm{S} 1$ & $1.502(3)$ \\
\hline $\mathrm{C} 10-\mathrm{H} 10 \mathrm{C}$ & 0.9900 & $\mathrm{O} 13-\mathrm{S} 1$ & $1.461(3)$ \\
\hline $\mathrm{C} 10-\mathrm{H} 10 \mathrm{D}$ & 0.9900 & $\mathrm{O} 14-\mathrm{S} 1$ & $1.448(4)$ \\
\hline $\mathrm{Cu} 1-\mathrm{N} 3$ & $1.915(4)$ & $\mathrm{O} 15-\mathrm{H} 15 \mathrm{~A}$ & $0.84(2)$ \\
\hline $\mathrm{Cu} 1-\mathrm{O} 1$ & $1.928(3)$ & O15-H15B & $0.84(2)$ \\
\hline $\mathrm{Cu} 1-\mathrm{O} 2$ & $1.969(3)$ & $\mathrm{O} 16-\mathrm{H} 16 \mathrm{~A}$ & $0.84(2)$ \\
\hline $\mathrm{Cu} 1-\mathrm{N} 4$ & $1.991(4)$ & $\mathrm{O} 16-\mathrm{H} 16 \mathrm{~B}$ & $0.84(2)$ \\
\hline $\mathrm{Cu} 1-\mathrm{O} 20$ & $2.601(4)$ & O17-H17A & $0.83(2)$ \\
\hline $\mathrm{Cu} 1-\mathrm{O} 21$ & $2.736(4)$ & O17-H17B & $0.83(2)$ \\
\hline $\mathrm{Cu} 2-\mathrm{N} 5$ & $1.900(4)$ & $\mathrm{O} 18-\mathrm{H} 18 \mathrm{~A}$ & $0.84(2)$ \\
\hline $\mathrm{Cu} 2-\mathrm{O} 3$ & $1.928(3)$ & $\mathrm{O} 18-\mathrm{H} 18 \mathrm{~B}$ & $0.83(2)$ \\
\hline $\mathrm{Cu} 2-\mathrm{O} 4$ & $1.936(3)$ & O19-H19A & $0.84(2)$ \\
\hline $\mathrm{Cu} 2-\mathrm{N} 6$ & $2.018(4)$ & O19-H19B & $0.84(2)$ \\
\hline $\mathrm{Cu} 2-\mathrm{O} 19$ & $2.409(10)$ & $\mathrm{O} 20-\mathrm{H} 20 \mathrm{~A}$ & $0.83(2)$ \\
\hline $\mathrm{Cu} 3-\mathrm{N} 7$ & $1.904(4)$ & $\mathrm{O} 20-\mathrm{H} 20 \mathrm{~B}$ & $0.83(2)$ \\
\hline $\mathrm{Cu} 3-\mathrm{O} 6$ & $1.944(3)$ & $\mathrm{O} 21-\mathrm{H} 21 \mathrm{~A}$ & $0.84(2)$ \\
\hline $\mathrm{Cu} 3-\mathrm{O} 5$ & $1.949(3)$ & $\mathrm{O} 21-\mathrm{H} 21 \mathrm{~B}$ & $0.83(2)$ \\
\hline $\mathrm{Cu} 3-\mathrm{N} 8$ & $2.014(4)$ & $\mathrm{O} 22-\mathrm{H} 22 \mathrm{~A}$ & $0.84(2)$ \\
\hline $\mathrm{Cu} 3-\mathrm{O} 16$ & $2.508(4)$ & $\mathrm{O} 22-\mathrm{H} 22 \mathrm{~B}$ & $0.84(2)$ \\
\hline $\mathrm{Cu} 4-\mathrm{N} 9$ & $1.894(4)$ & $\mathrm{O} 23-\mathrm{H} 23 \mathrm{~A}$ & $0.84(2)$ \\
\hline $\mathrm{Cu} 4-\mathrm{O} 8$ & $1.940(3)$ & $\mathrm{O} 23-\mathrm{H} 23 \mathrm{~B}$ & $0.85(2)$ \\
\hline $\mathrm{Cu} 4-\mathrm{O} 7$ & $1.947(3)$ & $\mathrm{O} 24-\mathrm{H} 24 \mathrm{~A}$ & $0.84(2)$ \\
\hline $\mathrm{Cu} 4-\mathrm{N} 10$ & $2.012(4)$ & $\mathrm{O} 24-\mathrm{H} 24 \mathrm{~B}$ & $0.84(2)$ \\
\hline $\mathrm{Cu} 4-\mathrm{O} 17$ & $2.481(4)$ & $\mathrm{O} 25-\mathrm{S} 2$ & $1.519(7)$ \\
\hline $\mathrm{Cu} 5-\mathrm{N} 1$ & $1.890(4)$ & $\mathrm{O} 26-\mathrm{S} 2$ & $1.401(8)$ \\
\hline $\mathrm{Cu} 5-\mathrm{O} 9$ & $1.943(3)$ & $\mathrm{O} 27-\mathrm{S} 2$ & $1.485(7)$ \\
\hline $\mathrm{Cu} 5-\mathrm{O} 10$ & $1.946(3)$ & $\mathrm{O} 28-\mathrm{S} 2$ & $1.458(8)$ \\
\hline $\mathrm{N} 1-\mathrm{C} 1-\mathrm{O} 2$ & $125.3(4)$ & $\mathrm{O} 5-\mathrm{Tb} 1-\mathrm{O} 11$ & $112.83(11)$ \\
\hline $\mathrm{N} 1-\mathrm{C} 1-\mathrm{C} 2$ & $114.2(4)$ & $\mathrm{O} 12-\mathrm{Tb} 1-\mathrm{O} 11$ & $57.34(11)$ \\
\hline $\mathrm{O} 2-\mathrm{C} 1-\mathrm{C} 2$ & $120.5(4)$ & $\mathrm{O} 9-\mathrm{Tb} 1-\mathrm{S} 1$ & $83.26(8)$ \\
\hline $\mathrm{N} 2-\mathrm{C} 2-\mathrm{C} 1$ & $110.0(4)$ & $\mathrm{O} 1-\mathrm{Tb} 1-\mathrm{S} 1$ & $102.84(8)$ \\
\hline $\mathrm{N} 2-\mathrm{C} 2-\mathrm{H} 2 \mathrm{C}$ & 109.7 & $\mathrm{O} 15-\mathrm{Tb} 1-\mathrm{S} 1$ & $174.96(8)$ \\
\hline
\end{tabular}




\begin{tabular}{|c|c|c|c|}
\hline $\mathrm{C} 1-\mathrm{C} 2-\mathrm{H} 2 \mathrm{C}$ & 109.7 & $\mathrm{O} 3-\mathrm{Tb} 1-\mathrm{S} 1$ & $96.74(9)$ \\
\hline $\mathrm{N} 2-\mathrm{C} 2-\mathrm{H} 2 \mathrm{D}$ & 109.7 & $\mathrm{O} 7-\mathrm{Tb} 1-\mathrm{S} 1$ & $101.43(8)$ \\
\hline $\mathrm{C} 1-\mathrm{C} 2-\mathrm{H} 2 \mathrm{D}$ & 109.7 & $\mathrm{O} 5-\mathrm{Tb} 1-\mathrm{S} 1$ & $101.06(8)$ \\
\hline $\mathrm{H} 2 \mathrm{C}-\mathrm{C} 2-\mathrm{H} 2 \mathrm{D}$ & 108.2 & $\mathrm{O} 12-\mathrm{Tb} 1-\mathrm{S} 1$ & $28.74(8)$ \\
\hline $\mathrm{N} 3-\mathrm{C} 3-\mathrm{O} 4$ & $123.0(4)$ & $\mathrm{O} 11-\mathrm{Tb} 1-\mathrm{S} 1$ & $28.77(8)$ \\
\hline $\mathrm{N} 3-\mathrm{C} 3-\mathrm{C} 4$ & $115.9(4)$ & $\mathrm{C} 1-\mathrm{N} 1-\mathrm{O} 1$ & $115.9(3)$ \\
\hline $\mathrm{O} 4-\mathrm{C} 3-\mathrm{C} 4$ & $121.1(4)$ & $\mathrm{C} 1-\mathrm{N} 1-\mathrm{Cu} 5$ & $119.5(3)$ \\
\hline $\mathrm{C} 3-\mathrm{C} 4-\mathrm{N} 4$ & $111.1(4)$ & $\mathrm{O} 1-\mathrm{N} 1-\mathrm{Cu} 5$ & $124.1(3)$ \\
\hline $\mathrm{C} 3-\mathrm{C} 4-\mathrm{H} 4 \mathrm{C}$ & 109.4 & $\mathrm{C} 2-\mathrm{N} 2-\mathrm{Cu} 5$ & $109.8(3)$ \\
\hline $\mathrm{N} 4-\mathrm{C} 4-\mathrm{H} 4 \mathrm{C}$ & 109.4 & $\mathrm{C} 2-\mathrm{N} 2-\mathrm{H} 2 \mathrm{~A}$ & 109.7 \\
\hline $\mathrm{C} 3-\mathrm{C} 4-\mathrm{H} 4 \mathrm{D}$ & 109.4 & $\mathrm{Cu} 5-\mathrm{N} 2-\mathrm{H} 2 \mathrm{~A}$ & 109.7 \\
\hline $\mathrm{N} 4-\mathrm{C} 4-\mathrm{H} 4 \mathrm{D}$ & 109.4 & $\mathrm{C} 2-\mathrm{N} 2-\mathrm{H} 2 \mathrm{~B}$ & 109.7 \\
\hline $\mathrm{H} 4 \mathrm{C}-\mathrm{C} 4-\mathrm{H} 4 \mathrm{D}$ & 108.0 & $\mathrm{Cu} 5-\mathrm{N} 2-\mathrm{H} 2 \mathrm{~B}$ & 109.7 \\
\hline $\mathrm{N} 5-\mathrm{C} 5-\mathrm{O} 6$ & $123.7(4)$ & $\mathrm{H} 2 \mathrm{~A}-\mathrm{N} 2-\mathrm{H} 2 \mathrm{~B}$ & 108.2 \\
\hline $\mathrm{N} 5-\mathrm{C} 5-\mathrm{C} 6$ & $116.0(4)$ & $\mathrm{C} 3-\mathrm{N} 3-\mathrm{O} 3$ & $115.1(4)$ \\
\hline $\mathrm{O} 6-\mathrm{C} 5-\mathrm{C} 6$ & $120.3(4)$ & $\mathrm{C} 3-\mathrm{N} 3-\mathrm{Cu} 1$ & $117.5(3)$ \\
\hline $\mathrm{N} 6-\mathrm{C} 6-\mathrm{C} 5$ & $110.5(4)$ & $\mathrm{O} 3-\mathrm{N} 3-\mathrm{Cu} 1$ & $125.5(3)$ \\
\hline $\mathrm{N} 6-\mathrm{C} 6-\mathrm{H} 6 \mathrm{C}$ & 109.5 & $\mathrm{C} 4-\mathrm{N} 4-\mathrm{Cu} 1$ & $110.6(3)$ \\
\hline $\mathrm{C} 5-\mathrm{C} 6-\mathrm{H} 6 \mathrm{C}$ & 109.5 & $\mathrm{C} 4-\mathrm{N} 4-\mathrm{H} 4 \mathrm{~A}$ & 109.5 \\
\hline $\mathrm{N} 6-\mathrm{C} 6-\mathrm{H} 6 \mathrm{D}$ & 109.5 & $\mathrm{Cu} 1-\mathrm{N} 4-\mathrm{H} 4 \mathrm{~A}$ & 109.5 \\
\hline $\mathrm{C} 5-\mathrm{C} 6-\mathrm{H} 6 \mathrm{D}$ & 109.5 & $\mathrm{C} 4-\mathrm{N} 4-\mathrm{H} 4 \mathrm{~B}$ & 109.5 \\
\hline $\mathrm{H} 6 \mathrm{C}-\mathrm{C} 6-\mathrm{H} 6 \mathrm{D}$ & 108.1 & $\mathrm{Cu} 1-\mathrm{N} 4-\mathrm{H} 4 \mathrm{~B}$ & 109.5 \\
\hline $\mathrm{N} 7-\mathrm{C} 7-\mathrm{O} 8$ & $124.1(4)$ & $\mathrm{H} 4 \mathrm{~A}-\mathrm{N} 4-\mathrm{H} 4 \mathrm{~B}$ & 108.1 \\
\hline $\mathrm{N} 7-\mathrm{C} 7-\mathrm{C} 8$ & $115.5(4)$ & $\mathrm{C} 5-\mathrm{N} 5-\mathrm{O} 5$ & $115.7(4)$ \\
\hline $\mathrm{O} 8-\mathrm{C} 7-\mathrm{C} 8$ & $120.4(4)$ & $\mathrm{C} 5-\mathrm{N} 5-\mathrm{Cu} 2$ & $118.6(3)$ \\
\hline $\mathrm{N} 8-\mathrm{C} 8-\mathrm{C} 7$ & $109.8(4)$ & $\mathrm{O} 5-\mathrm{N} 5-\mathrm{Cu} 2$ & $125.5(3)$ \\
\hline $\mathrm{N} 8-\mathrm{C} 8-\mathrm{H} 8 \mathrm{C}$ & 109.7 & $\mathrm{C} 6-\mathrm{N} 6-\mathrm{Cu} 2$ & $109.6(3)$ \\
\hline $\mathrm{C} 7-\mathrm{C} 8-\mathrm{H} 8 \mathrm{C}$ & 109.7 & C6-N6-H6A & 109.7 \\
\hline $\mathrm{N} 8-\mathrm{C} 8-\mathrm{H} 8 \mathrm{D}$ & 109.7 & $\mathrm{Cu} 2-\mathrm{N} 6-\mathrm{H} 6 \mathrm{~A}$ & 109.7 \\
\hline $\mathrm{C} 7-\mathrm{C} 8-\mathrm{H} 8 \mathrm{D}$ & 109.7 & $\mathrm{C} 6-\mathrm{N} 6-\mathrm{H} 6 \mathrm{~B}$ & 109.7 \\
\hline $\mathrm{H} 8 \mathrm{C}-\mathrm{C} 8-\mathrm{H} 8 \mathrm{D}$ & 108.2 & $\mathrm{Cu} 2-\mathrm{N} 6-\mathrm{H} 6 \mathrm{~B}$ & 109.7 \\
\hline $\mathrm{O} 10-\mathrm{C} 9-\mathrm{N} 9$ & $123.8(4)$ & $\mathrm{H} 6 \mathrm{~A}-\mathrm{N} 6-\mathrm{H} 6 \mathrm{~B}$ & 108.2 \\
\hline $\mathrm{O} 10-\mathrm{C} 9-\mathrm{C} 10$ & $121.2(4)$ & $\mathrm{C} 7-\mathrm{N} 7-\mathrm{O} 7$ & $116.2(4)$ \\
\hline $\mathrm{N} 9-\mathrm{C} 9-\mathrm{C} 10$ & $115.0(4)$ & $\mathrm{C} 7-\mathrm{N} 7-\mathrm{Cu} 3$ & $119.0(3)$ \\
\hline $\mathrm{N} 10-\mathrm{C} 10-\mathrm{C} 9$ & $111.3(4)$ & $\mathrm{O} 7-\mathrm{N} 7-\mathrm{Cu} 3$ & $124.6(3)$ \\
\hline $\mathrm{N} 10-\mathrm{C} 10-\mathrm{H} 10 \mathrm{C}$ & 109.4 & $\mathrm{C} 8-\mathrm{N} 8-\mathrm{Cu} 3$ & $109.7(3)$ \\
\hline $\mathrm{C} 9-\mathrm{C} 10-\mathrm{H} 10 \mathrm{C}$ & 109.4 & $\mathrm{C} 8-\mathrm{N} 8-\mathrm{H} 8 \mathrm{~A}$ & 109.7 \\
\hline $\mathrm{N} 10-\mathrm{C} 10-\mathrm{H} 10 \mathrm{D}$ & 109.4 & $\mathrm{Cu} 3-\mathrm{N} 8-\mathrm{H} 8 \mathrm{~A}$ & 109.7 \\
\hline $\mathrm{C} 9-\mathrm{C} 10-\mathrm{H} 10 \mathrm{D}$ & 109.4 & $\mathrm{C} 8-\mathrm{N} 8-\mathrm{H} 8 \mathrm{~B}$ & 109.7 \\
\hline $\mathrm{H} 10 \mathrm{C}-\mathrm{C} 10-\mathrm{H} 10 \mathrm{D}$ & 108.0 & $\mathrm{Cu} 3-\mathrm{N} 8-\mathrm{H} 8 \mathrm{~B}$ & 109.7 \\
\hline $\mathrm{N} 3-\mathrm{Cu} 1-\mathrm{O} 1$ & $90.36(14)$ & $\mathrm{H} 8 \mathrm{~A}-\mathrm{N} 8-\mathrm{H} 8 \mathrm{~B}$ & 108.2 \\
\hline $\mathrm{N} 3-\mathrm{Cu} 1-\mathrm{O} 2$ & $175.68(15)$ & $\mathrm{C} 9-\mathrm{N} 9-\mathrm{O} 9$ & $116.1(4)$ \\
\hline $\mathrm{O} 1-\mathrm{Cu} 1-\mathrm{O} 2$ & $86.12(13)$ & $\mathrm{C} 9-\mathrm{N} 9-\mathrm{Cu} 4$ & $119.1(3)$ \\
\hline $\mathrm{N} 3-\mathrm{Cu} 1-\mathrm{N} 4$ & $83.85(16)$ & $\mathrm{O} 9-\mathrm{N} 9-\mathrm{Cu} 4$ & $124.1(3)$ \\
\hline $\mathrm{O} 1-\mathrm{Cu} 1-\mathrm{N} 4$ & $173.91(15)$ & $\mathrm{C} 10-\mathrm{N} 10-\mathrm{Cu} 4$ & $109.9(3)$ \\
\hline $\mathrm{O} 2-\mathrm{Cu} 1-\mathrm{N} 4$ & $99.57(15)$ & $\mathrm{C} 10-\mathrm{N} 10-\mathrm{H} 10 \mathrm{~A}$ & 109.7 \\
\hline $\mathrm{N} 3-\mathrm{Cu} 1-\mathrm{O} 20$ & $89.10(15)$ & $\mathrm{Cu} 4-\mathrm{N} 10-\mathrm{H} 10 \mathrm{~A}$ & 109.7 \\
\hline $\mathrm{O} 1-\mathrm{Cu} 1-\mathrm{O} 20$ & $85.07(13)$ & $\mathrm{C} 10-\mathrm{N} 10-\mathrm{H} 10 \mathrm{~B}$ & 109.7 \\
\hline
\end{tabular}




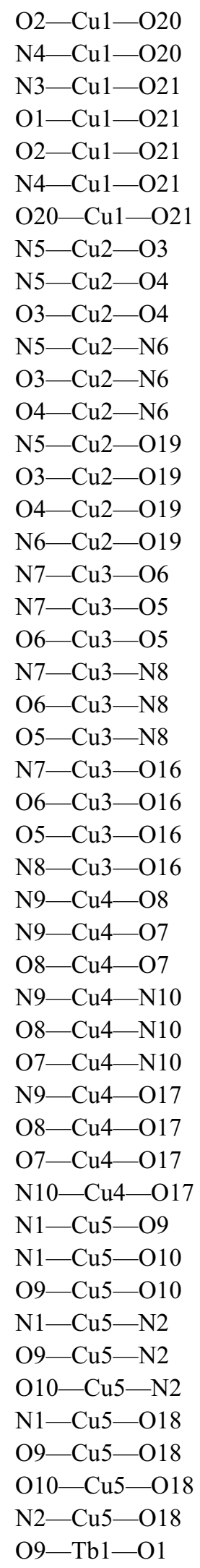

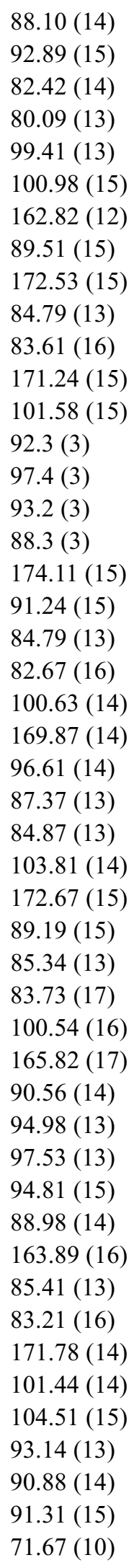

\begin{tabular}{|c|c|}
\hline $\mathrm{Cu} 4-\mathrm{N} 10-\mathrm{H} 10 \mathrm{~B}$ & 109.7 \\
\hline $\mathrm{H} 10 \mathrm{~A}-\mathrm{N} 10-\mathrm{H} 10 \mathrm{~B}$ & 108.2 \\
\hline $\mathrm{N} 1-\mathrm{O} 1-\mathrm{Cu} 1$ & $106.2(2)$ \\
\hline $\mathrm{N} 1-\mathrm{O} 1-\mathrm{Tb} 1$ & $125.6(2)$ \\
\hline $\mathrm{Cu} 1-\mathrm{O} 1-\mathrm{Tb} 1$ & $126.24(14)$ \\
\hline $\mathrm{C} 1-\mathrm{O} 2-\mathrm{Cu} 1$ & $104.0(3)$ \\
\hline $\mathrm{N} 3-\mathrm{O} 3-\mathrm{Cu} 2$ & $108.6(2)$ \\
\hline $\mathrm{N} 3-\mathrm{O} 3-\mathrm{Tb} 1$ & $122.4(2)$ \\
\hline $\mathrm{Cu} 2-\mathrm{O} 3-\mathrm{Tb} 1$ & $128.77(15)$ \\
\hline $\mathrm{C} 3-\mathrm{O} 4-\mathrm{Cu} 2$ & $107.7(3)$ \\
\hline $\mathrm{N} 5-\mathrm{O} 5-\mathrm{Cu} 3$ & $107.1(2)$ \\
\hline $\mathrm{N} 5-\mathrm{O} 5-\mathrm{Tb} 1$ & $124.1(2)$ \\
\hline $\mathrm{Cu} 3-\mathrm{O} 5-\mathrm{Tb} 1$ & $123.88(13)$ \\
\hline $\mathrm{C} 5-\mathrm{O} 6-\mathrm{Cu} 3$ & $107.0(3)$ \\
\hline $\mathrm{N} 7-\mathrm{O} 7-\mathrm{Cu} 4$ & $107.2(2)$ \\
\hline $\mathrm{N} 7-\mathrm{O} 7-\mathrm{Tb} 1$ & $124.7(2)$ \\
\hline $\mathrm{Cu} 4-\mathrm{O} 7-\mathrm{Tb} 1$ & $125.89(14)$ \\
\hline $\mathrm{C} 7-\mathrm{O} 8-\mathrm{Cu} 4$ & $106.9(3)$ \\
\hline $\mathrm{N} 9-\mathrm{O} 9-\mathrm{Cu} 5$ & $107.2(2)$ \\
\hline N9-O9-Tb1 & $126.2(2)$ \\
\hline $\mathrm{Cu} 5-\mathrm{O} 9-\mathrm{Tb} 1$ & $126.55(14)$ \\
\hline $\mathrm{C} 9-\mathrm{O} 10-\mathrm{Cu} 5$ & $107.4(3)$ \\
\hline $\mathrm{S} 1-\mathrm{O} 11-\mathrm{Tb} 1$ & $99.41(17)$ \\
\hline $\mathrm{S} 1-\mathrm{O} 12-\mathrm{Tb} 1$ & $100.02(16)$ \\
\hline $\mathrm{Tb} 1-\mathrm{O} 15-\mathrm{H} 15 \mathrm{~A}$ & $121(4)$ \\
\hline $\mathrm{Tb} 1-\mathrm{O} 15-\mathrm{H} 15 \mathrm{~B}$ & $118(4)$ \\
\hline $\mathrm{H} 15 \mathrm{~A}-\mathrm{O} 15-\mathrm{H} 15 \mathrm{~B}$ & $107(6)$ \\
\hline $\mathrm{Cu} 3-\mathrm{O} 16-\mathrm{H} 16 \mathrm{~A}$ & $122(5)$ \\
\hline $\mathrm{Cu} 3-\mathrm{O} 16-\mathrm{H} 16 \mathrm{~B}$ & $114(5)$ \\
\hline $\mathrm{H} 16 \mathrm{~A}-\mathrm{O} 16-\mathrm{H} 16 \mathrm{~B}$ & $102(7)$ \\
\hline $\mathrm{Cu} 4-\mathrm{O} 17-\mathrm{H} 17 \mathrm{~A}$ & $92(5)$ \\
\hline $\mathrm{Cu} 4-\mathrm{O} 17-\mathrm{H} 17 \mathrm{~B}$ & $111(5)$ \\
\hline $\mathrm{H} 17 \mathrm{~A}-\mathrm{O} 17-\mathrm{H} 17 \mathrm{~B}$ & $103(6)$ \\
\hline $\mathrm{Cu} 5-\mathrm{O} 18-\mathrm{H} 18 \mathrm{~A}$ & $120(5)$ \\
\hline $\mathrm{Cu} 5-\mathrm{O} 18-\mathrm{H} 18 \mathrm{~B}$ & $115(5)$ \\
\hline $\mathrm{H} 18 \mathrm{~A}-\mathrm{O} 18-\mathrm{H} 18 \mathrm{~B}$ & $107(7)$ \\
\hline $\mathrm{Cu} 2-\mathrm{O} 19-\mathrm{H} 19 \mathrm{~A}$ & $99(10)$ \\
\hline $\mathrm{Cu} 2-\mathrm{O} 19-\mathrm{H} 19 \mathrm{~B}$ & $113(10)$ \\
\hline H19A-O19-H19B & $135(10)$ \\
\hline $\mathrm{Cu} 1-\mathrm{O} 20-\mathrm{H} 20 \mathrm{~A}$ & $131(5)$ \\
\hline $\mathrm{Cu} 1-\mathrm{O} 20-\mathrm{H} 20 \mathrm{~B}$ & $95(5)$ \\
\hline $\mathrm{H} 20 \mathrm{~A}-\mathrm{O} 20-\mathrm{H} 20 \mathrm{~B}$ & $93(7)$ \\
\hline $\mathrm{Cu} 1-\mathrm{O} 21-\mathrm{H} 21 \mathrm{~A}$ & $124(5)$ \\
\hline $\mathrm{Cu} 1-\mathrm{O} 21-\mathrm{H} 21 \mathrm{~B}$ & $110(5)$ \\
\hline $\mathrm{H} 21 \mathrm{~A}-\mathrm{O} 21-\mathrm{H} 21 \mathrm{~B}$ & $101(7)$ \\
\hline $\mathrm{H} 22 \mathrm{~A}-\mathrm{O} 22-\mathrm{H} 22 \mathrm{~B}$ & $113(8)$ \\
\hline $\mathrm{H} 23 \mathrm{~A}-\mathrm{O} 23-\mathrm{H} 23 \mathrm{~B}$ & $105(3)$ \\
\hline $\mathrm{H} 24 \mathrm{~A}-\mathrm{O} 24-\mathrm{H} 24 \mathrm{~B}$ & $108(3)$ \\
\hline
\end{tabular}




\begin{tabular}{|c|c|c|c|}
\hline $\mathrm{O} 9-\mathrm{Tb} 1-\mathrm{O} 15$ & $100.80(11)$ & $\mathrm{O} 14-\mathrm{S} 1-\mathrm{O} 13$ & $110.9(2)$ \\
\hline $\mathrm{O} 1-\mathrm{Tb} 1-\mathrm{O} 15$ & $75.87(11)$ & $\mathrm{O} 14-\mathrm{S} 1-\mathrm{O} 11$ & $111.0(2)$ \\
\hline $\mathrm{O} 9-\mathrm{Tb} 1-\mathrm{O} 3$ & $144.63(11)$ & $\mathrm{O} 13-\mathrm{S} 1-\mathrm{O} 11$ & $111.6(2)$ \\
\hline $\mathrm{O} 1-\mathrm{Tb} 1-\mathrm{O} 3$ & $73.91(10)$ & $\mathrm{O} 14-\mathrm{S} 1-\mathrm{O} 12$ & $110.1(2)$ \\
\hline $\mathrm{O} 15-\mathrm{Tb} 1-\mathrm{O} 3$ & $78.22(11)$ & $\mathrm{O} 13-\mathrm{S} 1-\mathrm{O} 12$ & $110.3(2)$ \\
\hline $\mathrm{O} 9-\mathrm{Tb} 1-\mathrm{O} 7$ & $70.65(10)$ & $\mathrm{O} 11-\mathrm{S} 1-\mathrm{O} 12$ & $102.68(19)$ \\
\hline $\mathrm{O} 1-\mathrm{Tb} 1-\mathrm{O} 7$ & $131.81(10)$ & $\mathrm{O} 14-\mathrm{S} 1-\mathrm{Tb} 1$ & $118.92(16)$ \\
\hline $\mathrm{O} 15-\mathrm{Tb} 1-\mathrm{O} 7$ & $82.82(11)$ & $\mathrm{O} 13-\mathrm{S} 1-\mathrm{Tb} 1$ & $130.15(15)$ \\
\hline $\mathrm{O} 3-\mathrm{Tb} 1-\mathrm{O} 7$ & $142.47(10)$ & $\mathrm{O} 11-\mathrm{S} 1-\mathrm{Tb} 1$ & $51.82(13)$ \\
\hline $\mathrm{O} 9-\mathrm{Tb} 1-\mathrm{O} 5$ & $143.39(10)$ & $\mathrm{O} 12-\mathrm{S} 1-\mathrm{Tb} 1$ & $51.24(13)$ \\
\hline $\mathrm{O} 1-\mathrm{Tb} 1-\mathrm{O} 5$ & $139.79(10)$ & $\mathrm{O} 26^{\mathrm{i}}-\mathrm{S} 2-\mathrm{O} 26$ & 180.0 \\
\hline $\mathrm{O} 15-\mathrm{Tb} 1-\mathrm{O} 5$ & $77.50(11)$ & $\mathrm{O} 26^{\mathrm{i}}-\mathrm{S} 2-\mathrm{O} 28^{\mathrm{i}}$ & $114.8(5)$ \\
\hline $\mathrm{O} 3-\mathrm{Tb} 1-\mathrm{O} 5$ & $71.55(10)$ & $\mathrm{O} 26-\mathrm{S} 2-\mathrm{O} 28^{\mathrm{i}}$ & $65.2(5)$ \\
\hline $\mathrm{O} 7-\mathrm{Tb} 1-\mathrm{O} 5$ & $72.88(10)$ & $\mathrm{O} 26^{\mathrm{i}}-\mathrm{S} 2-\mathrm{O} 28$ & $65.2(5)$ \\
\hline $\mathrm{O} 9-\mathrm{Tb} 1-\mathrm{O} 12$ & $83.74(11)$ & $\mathrm{O} 26-\mathrm{S} 2-\mathrm{O} 28$ & $114.8(5)$ \\
\hline $\mathrm{O} 1-\mathrm{Tb} 1-\mathrm{O} 12$ & $129.16(11)$ & $\mathrm{O} 28^{\mathrm{i}}-\mathrm{S} 2-\mathrm{O} 28$ & $180.0(5)$ \\
\hline $\mathrm{O} 15-\mathrm{Tb} 1-\mathrm{O} 12$ & $153.99(11)$ & $\mathrm{O} 26^{\mathrm{i}}-\mathrm{S} 2-\mathrm{O} 27$ & $68.7(5)$ \\
\hline $\mathrm{O} 3-\mathrm{Tb} 1-\mathrm{O} 12$ & $112.70(11)$ & $\mathrm{O} 26-\mathrm{S} 2-\mathrm{O} 27$ & $111.3(5)$ \\
\hline $\mathrm{O} 7-\mathrm{Tb} 1-\mathrm{O} 12$ & $74.50(11)$ & $\mathrm{O} 28^{\mathrm{i}}-\mathrm{S} 2-\mathrm{O} 27$ & $70.8(5)$ \\
\hline $\mathrm{O} 5-\mathrm{Tb} 1-\mathrm{O} 12$ & $83.70(11)$ & $\mathrm{O} 28-\mathrm{S} 2-\mathrm{O} 27$ & $109.2(5)$ \\
\hline $\mathrm{O} 9-\mathrm{Tb} 1-\mathrm{O} 11$ & $88.43(11)$ & $\mathrm{O} 26^{\mathrm{i}}-\mathrm{S} 2-\mathrm{O} 25$ & $69.6(5)$ \\
\hline $\mathrm{O} 1-\mathrm{Tb} 1-\mathrm{O} 11$ & $77.71(11)$ & $\mathrm{O} 26-\mathrm{S} 2-\mathrm{O} 25$ & $110.4(5)$ \\
\hline $\mathrm{O} 15-\mathrm{Tb} 1-\mathrm{O} 11$ & $147.49(12)$ & $\mathrm{O} 28^{\mathrm{i}}-\mathrm{S} 2-\mathrm{O} 25$ & $73.6(5)$ \\
\hline $\mathrm{O} 3-\mathrm{Tb} 1-\mathrm{O} 11$ & $76.51(12)$ & $\mathrm{O} 28-\mathrm{S} 2-\mathrm{O} 25$ & $106.4(5)$ \\
\hline $\mathrm{O} 7-\mathrm{Tb} 1-\mathrm{O} 11$ & $129.39(11)$ & $\mathrm{O} 27-\mathrm{S} 2-\mathrm{O} 25$ & $104.2(5)$ \\
\hline $\mathrm{N} 1-\mathrm{C} 1-\mathrm{C} 2-\mathrm{N} 2$ & $18.5(6)$ & $\mathrm{O} 10-\mathrm{C} 9-\mathrm{N} 9-\mathrm{Cu} 4$ & $-173.1(3)$ \\
\hline $\mathrm{O} 2-\mathrm{C} 1-\mathrm{C} 2-\mathrm{N} 2$ & $-161.5(4)$ & $\mathrm{C} 10-\mathrm{C} 9-\mathrm{N} 9-\mathrm{Cu} 4$ & $6.7(5)$ \\
\hline $\mathrm{N} 3-\mathrm{C} 3-\mathrm{C} 4-\mathrm{N} 4$ & $-9.9(6)$ & $\mathrm{O} 7-\mathrm{Cu} 4-\mathrm{N} 9-\mathrm{C} 9$ & $167.0(4)$ \\
\hline $\mathrm{O} 4-\mathrm{C} 3-\mathrm{C} 4-\mathrm{N} 4$ & $168.5(4)$ & $\mathrm{N} 10-\mathrm{Cu} 4-\mathrm{N} 9-\mathrm{C} 9$ & $-0.7(4)$ \\
\hline $\mathrm{N} 5-\mathrm{C} 5-\mathrm{C} 6-\mathrm{N} 6$ & $5.8(6)$ & $\mathrm{O} 17-\mathrm{Cu} 4-\mathrm{N} 9-\mathrm{C} 9$ & $-95.5(3)$ \\
\hline $\mathrm{O} 6-\mathrm{C} 5-\mathrm{C} 6-\mathrm{N} 6$ & $-176.2(4)$ & $\mathrm{O} 7-\mathrm{Cu} 4-\mathrm{N} 9-\mathrm{O} 9$ & $-2.8(3)$ \\
\hline $\mathrm{N} 7-\mathrm{C} 7-\mathrm{C} 8-\mathrm{N} 8$ & $-10.1(5)$ & $\mathrm{N} 10-\mathrm{Cu} 4-\mathrm{N} 9-\mathrm{O} 9$ & $-170.5(3)$ \\
\hline $\mathrm{O} 8-\mathrm{C} 7-\mathrm{C} 8-\mathrm{N} 8$ & $170.5(4)$ & $\mathrm{O} 17-\mathrm{Cu} 4-\mathrm{N} 9-\mathrm{O} 9$ & $94.8(3)$ \\
\hline $\mathrm{O} 10-\mathrm{C} 9-\mathrm{C} 10-\mathrm{N} 10$ & $168.9(4)$ & $\mathrm{C} 9-\mathrm{C} 10-\mathrm{N} 10-\mathrm{Cu} 4$ & $9.8(5)$ \\
\hline $\mathrm{N} 9-\mathrm{C} 9-\mathrm{C} 10-\mathrm{N} 10$ & $-10.9(6)$ & $\mathrm{C} 1-\mathrm{N} 1-\mathrm{O} 1-\mathrm{Cu} 1$ & $11.7(4)$ \\
\hline $\mathrm{O} 2-\mathrm{C} 1-\mathrm{N} 1-\mathrm{O} 1$ & $-0.6(6)$ & $\mathrm{Cu} 5-\mathrm{N} 1-\mathrm{O} 1-\mathrm{Cu} 1$ & $-159.6(2)$ \\
\hline $\mathrm{C} 2-\mathrm{C} 1-\mathrm{N} 1-\mathrm{O} 1$ & $179.4(4)$ & $\mathrm{C} 1-\mathrm{N} 1-\mathrm{O} 1-\mathrm{Tb} 1$ & $176.5(3)$ \\
\hline $\mathrm{O} 2-\mathrm{C} 1-\mathrm{N} 1-\mathrm{Cu} 5$ & $171.2(3)$ & $\mathrm{Cu} 5-\mathrm{N} 1-\mathrm{O} 1-\mathrm{Tb} 1$ & $5.2(5)$ \\
\hline $\mathrm{C} 2-\mathrm{C} 1-\mathrm{N} 1-\mathrm{Cu} 5$ & $-8.8(5)$ & $\mathrm{N} 1-\mathrm{C} 1-\mathrm{O} 2-\mathrm{Cu} 1$ & $-10.6(5)$ \\
\hline $\mathrm{O} 9-\mathrm{Cu} 5-\mathrm{N} 1-\mathrm{C} 1$ & $175.3(4)$ & $\mathrm{C} 2-\mathrm{C} 1-\mathrm{O} 2-\mathrm{Cu} 1$ & $169.4(3)$ \\
\hline $\mathrm{O} 10-\mathrm{Cu} 5-\mathrm{N} 1-\mathrm{C} 1$ & $105.8(6)$ & $\mathrm{C} 3-\mathrm{N} 3-\mathrm{O} 3-\mathrm{Cu} 2$ & $-5.7(4)$ \\
\hline $\mathrm{N} 2-\mathrm{Cu} 5-\mathrm{N} 1-\mathrm{C} 1$ & $-2.1(4)$ & $\mathrm{Cu} 1-\mathrm{N} 3-\mathrm{O} 3-\mathrm{Cu} 2$ & $158.1(2)$ \\
\hline $\mathrm{O} 18-\mathrm{Cu} 5-\mathrm{N} 1-\mathrm{C} 1$ & $-91.7(4)$ & $\mathrm{C} 3-\mathrm{N} 3-\mathrm{O} 3-\mathrm{Tb} 1$ & $179.5(3)$ \\
\hline $\mathrm{O} 9-\mathrm{Cu} 5-\mathrm{N} 1-\mathrm{O} 1$ & $-13.6(3)$ & $\mathrm{Cu} 1-\mathrm{N} 3-\mathrm{O} 3-\mathrm{Tb} 1$ & $-16.7(5)$ \\
\hline $\mathrm{O} 10-\mathrm{Cu} 5-\mathrm{N} 1-\mathrm{O} 1$ & $-83.1(6)$ & $\mathrm{N} 3-\mathrm{C} 3-\mathrm{O} 4-\mathrm{Cu} 2$ & $7.1(5)$ \\
\hline $\mathrm{N} 2-\mathrm{Cu} 5-\mathrm{N} 1-\mathrm{O} 1$ & $169.0(3)$ & $\mathrm{C} 4-\mathrm{C} 3-\mathrm{O} 4-\mathrm{Cu} 2$ & $-171.2(4)$ \\
\hline $\mathrm{O} 18-\mathrm{Cu} 5-\mathrm{N} 1-\mathrm{O} 1$ & $79.4(3)$ & $\mathrm{C} 5-\mathrm{N} 5-\mathrm{O} 5-\mathrm{Cu} 3$ & $-9.7(4)$ \\
\hline
\end{tabular}




$\begin{array}{ll}\mathrm{C} 1-\mathrm{C} 2-\mathrm{N} 2-\mathrm{Cu} 5 & -19.1(5) \\ \mathrm{O} 4-\mathrm{C} 3-\mathrm{N} 3-\mathrm{O} 3 & -1.0(6) \\ \mathrm{C} 4-\mathrm{C} 3-\mathrm{N} 3-\mathrm{O} 3 & 177.4(4) \\ \mathrm{O} 4-\mathrm{C} 3-\mathrm{N} 3-\mathrm{Cu} 1 & -166.2(3) \\ \mathrm{C} 4-\mathrm{C} 3-\mathrm{N} 3-\mathrm{Cu} 1 & 12.2(5) \\ \mathrm{C} 3-\mathrm{C} 4-\mathrm{N} 4-\mathrm{Cu} 1 & 3.3(5) \\ \mathrm{O} 6-\mathrm{C} 5-\mathrm{N} 5-\mathrm{O} 5 & 0.7(6) \\ \mathrm{C} 6-\mathrm{C} 5-\mathrm{N} 5-\mathrm{O} 5 & 178.6(4) \\ \mathrm{O} 6-\mathrm{C} 5-\mathrm{N} 5-\mathrm{Cu} 2 & -173.6(3) \\ \mathrm{C} 6-\mathrm{C} 5-\mathrm{N} 5-\mathrm{Cu} 2 & 4.4(5) \\ \mathrm{O} 3-\mathrm{Cu} 2-\mathrm{N} 5-\mathrm{C} 5 & 165.2(4) \\ \mathrm{N} 6-\mathrm{Cu} 2-\mathrm{N} 5-\mathrm{C} 5 & -9.4(3) \\ \mathrm{O} 19-\mathrm{Cu} 2-\mathrm{N} 5-\mathrm{C} 5 & -97.4(4) \\ \mathrm{O} 3-\mathrm{Cu} 2-\mathrm{N} 5-\mathrm{O} 5 & -8.4(3) \\ \mathrm{N} 6-\mathrm{Cu} 2-\mathrm{N} 5-\mathrm{O} 5 & 177.0(3) \\ \mathrm{O} 19-\mathrm{Cu} 2-\mathrm{N} 5-\mathrm{O} 5 & 88.9(4) \\ \mathrm{C} 5-\mathrm{C} 6-\mathrm{N} 6-\mathrm{Cu} 2 & -12.1(5) \\ \mathrm{O} 8-\mathrm{C} 7-\mathrm{N} 7-\mathrm{O} 7 & 0.3(6) \\ \mathrm{C} 8-\mathrm{C} 7-\mathrm{N} 7-\mathrm{O} 7 & -179.1(4) \\ \mathrm{O} 8-\mathrm{C} 7-\mathrm{N} 7-\mathrm{Cu} 3 & 175.5(3) \\ \mathrm{C} 8-\mathrm{C} 7-\mathrm{N} 7-\mathrm{Cu} 3 & -3.9(5) \\ \mathrm{C} 7-\mathrm{C} 8-\mathrm{N} 8-\mathrm{Cu} 3 & 18.0(4) \\ \mathrm{O} 10-\mathrm{C} 9-\mathrm{N} 9-\mathrm{O} 9 & -2.5(6) \\ \mathrm{C} 10-\mathrm{C} 9-\mathrm{N} 9-\mathrm{O} 9 & 177.3(4) \\ \end{array}$

$\mathrm{Cu} 2-\mathrm{N} 5-\mathrm{O} 5-\mathrm{Cu} 3$

$\mathrm{C} 5-\mathrm{N} 5-\mathrm{O} 5-\mathrm{Tb} 1$

$\mathrm{Cu} 2-\mathrm{N} 5-\mathrm{O} 5-\mathrm{Tb} 1$

$\mathrm{N} 5-\mathrm{C} 5-\mathrm{O} 6-\mathrm{Cu} 3$

$\mathrm{C} 6-\mathrm{C} 5-\mathrm{O} 6-\mathrm{Cu} 3$

$\mathrm{C} 7-\mathrm{N} 7-\mathrm{O} 7-\mathrm{Cu} 4$

$\mathrm{Cu} 3-\mathrm{N} 7-\mathrm{O} 7-\mathrm{Cu} 4$

C7-N7-O7- $\mathrm{Tb} 1$

$\mathrm{Cu} 3-\mathrm{N} 7-\mathrm{O} 7-\mathrm{Tb} 1$

$\mathrm{N} 7-\mathrm{C} 7-\mathrm{O} 8-\mathrm{Cu} 4$

$\mathrm{C} 8-\mathrm{C} 7-\mathrm{O} 8-\mathrm{Cu} 4$

$\mathrm{C} 9-\mathrm{N} 9-\mathrm{O} 9-\mathrm{Cu} 5$

$\mathrm{Cu} 4-\mathrm{N} 9-\mathrm{O} 9-\mathrm{Cu} 5$

C9-N9-O9- $\mathrm{Tb} 1$

$\mathrm{Cu} 4-\mathrm{N} 9-\mathrm{O} 9-\mathrm{Tb} 1$

N9-C9-O10- $\mathrm{Cu} 5$

$\mathrm{C} 10-\mathrm{C} 9-\mathrm{O} 10-\mathrm{Cu} 5$

$\mathrm{Tb} 1-\mathrm{O} 11-\mathrm{S} 1-\mathrm{O} 14$

$\mathrm{Tb} 1-\mathrm{O} 11-\mathrm{S} 1-\mathrm{O} 13$

$\mathrm{Tb} 1-\mathrm{O} 11-\mathrm{S} 1-\mathrm{O} 12$

$\mathrm{Tb} 1-\mathrm{O} 12-\mathrm{S} 1-\mathrm{O} 14$

$\mathrm{Tb} 1-\mathrm{O} 12-\mathrm{S} 1-\mathrm{O} 13$

$\mathrm{Tb} 1-\mathrm{O} 12-\mathrm{S} 1-\mathrm{O} 11$
$164.1(2)$

$-165.6(3)$

$8.2(4)$

$8.8(5)$

$-169.1(3)$

$3.2(4)$

$-171.6(2)$

$167.2(3)$

$-7.7(5)$

$-3.7(5)$

175.7 (3)

$-0.7(4)$

$169.3(2)$

$177.4(3)$

$-12.5(5)$

$4.2(5)$

$-175.6(4)$

$-110.9(2)$

$124.81(18)$

$6.7(2)$

$111.5(2)$

$-125.78(18)$

-6.7 (2)

Symmetry code: (i) $-x,-y,-z+1$.

Hydrogen-bond geometry $\left(\AA,{ }^{\circ}\right)$

\begin{tabular}{|c|c|c|c|c|}
\hline$D-\mathrm{H} \cdots A$ & $D-\mathrm{H}$ & $\mathrm{H} \cdots A$ & $D \cdots A$ & $D-\mathrm{H} \cdots A$ \\
\hline $\mathrm{O} 24-\mathrm{H} 24 B \cdots \mathrm{O} 8$ & $0.84(2)$ & $2.01(3)$ & $2.807(5)$ & $159(7)$ \\
\hline $\mathrm{O} 24-\mathrm{H} 24 A \cdots \mathrm{O} 11^{\mathrm{ii}}$ & $0.84(2)$ & $2.21(3)$ & $3.015(5)$ & $162(7)$ \\
\hline $\mathrm{O} 23-\mathrm{H} 23 B \cdots \mathrm{O} 13^{\mathrm{iii}}$ & $0.85(2)$ & $2.02(3)$ & $2.853(5)$ & $166(6)$ \\
\hline $\mathrm{O} 23-\mathrm{H} 23 A \cdots \mathrm{O} 4^{\mathrm{ii}}$ & $0.84(2)$ & $1.89(2)$ & $2.734(5)$ & $176(7)$ \\
\hline $\mathrm{O} 22-\mathrm{H} 22 B \cdots \mathrm{O} 23$ & $0.84(2)$ & $1.89(3)$ & $2.701(6)$ & $162(8)$ \\
\hline $\mathrm{O} 22-\mathrm{H} 22 A \cdots \mathrm{O} 26^{\mathrm{iv}}$ & $0.84(2)$ & $2.18(4)$ & $2.968(9)$ & $155(8)$ \\
\hline $\mathrm{O} 22-\mathrm{H} 22 A \cdots \mathrm{O} 28^{\mathrm{iii}}$ & $0.84(2)$ & $1.92(3)$ & $2.733(9)$ & $161(8)$ \\
\hline $\mathrm{O} 21-\mathrm{H} 21 B \cdots \mathrm{O} 10^{\mathrm{iv}}$ & $0.83(2)$ & $1.91(3)$ & $2.728(5)$ & $165(8)$ \\
\hline $\mathrm{O} 21-\mathrm{H} 21 A^{\cdots} \mathrm{O} 18^{\mathrm{v}}$ & $0.84(2)$ & $1.94(3)$ & $2.765(5)$ & $167(7)$ \\
\hline $\mathrm{O} 20-\mathrm{H} 20 B \cdots \mathrm{O} 11$ & $0.83(2)$ & $2.14(3)$ & $2.960(5)$ & $168(7)$ \\
\hline $\mathrm{O} 20-\mathrm{H} 20 A \cdots \mathrm{O} 26^{\mathrm{i}}$ & $0.83(2)$ & $2.09(3)$ & $2.916(9)$ & $170(7)$ \\
\hline $\mathrm{O} 20-\mathrm{H} 20 A \cdots \mathrm{O} 25$ & $0.83(2)$ & $2.02(5)$ & $2.719(9)$ & $142(7)$ \\
\hline $\mathrm{O} 19-\mathrm{H} 19 B \cdots \mathrm{O} 24^{\mathrm{vi}}$ & $0.84(2)$ & $2.07(9)$ & $2.866(11)$ & $157(22)$ \\
\hline $\mathrm{O} 19-\mathrm{H} 19 A \cdots \mathrm{O} 24^{\text {vii }}$ & $0.84(2)$ & $1.72(7)$ & $2.535(12)$ & $162(21)$ \\
\hline $\mathrm{O} 18-\mathrm{H} 18 B \cdots \mathrm{O} 14$ & $0.83(2)$ & $1.90(2)$ & $2.732(5)$ & $173(7)$ \\
\hline $\mathrm{O} 18-\mathrm{H} 18 A \cdots \mathrm{O} 26^{\mathrm{i}}$ & $0.84(2)$ & $2.04(3)$ & $2.857(9)$ & $163(7)$ \\
\hline $\mathrm{O} 18-\mathrm{H} 18 A \cdots \mathrm{O} 27$ & $0.84(2)$ & $1.91(4)$ & $2.648(9)$ & $146(6)$ \\
\hline $\mathrm{O} 17-\mathrm{H} 17 B \cdots \mathrm{O}^{\mathrm{vi}}$ & $0.83(2)$ & $1.90(2)$ & $2.730(5)$ & $176(7)$ \\
\hline
\end{tabular}


supporting information

\begin{tabular}{lllll}
$\mathrm{O} 17-\mathrm{H} 17 A \cdots \mathrm{O} 12$ & $0.83(2)$ & $2.10(3)$ & $2.905(5)$ & $163(6)$ \\
$\mathrm{O} 16-\mathrm{H} 16 B \cdots \mathrm{O} 22$ & $0.84(2)$ & $1.89(2)$ & $2.721(6)$ & $173(7)$ \\
$\mathrm{O} 16-\mathrm{H} 16 A \cdots \mathrm{O} 17^{\mathrm{v}}$ & $0.84(2)$ & $1.95(2)$ & $2.784(5)$ & $172(7)$ \\
$\mathrm{O} 15-\mathrm{H} 15 B \cdots \mathrm{O} 16$ & $0.84(2)$ & $1.86(2)$ & $2.692(5)$ & $170(6)$ \\
$\mathrm{O} 15-\mathrm{H} 15 A \cdots \mathrm{O} 21$ & $0.84(2)$ & $1.85(3)$ & $2.668(5)$ & $166(6)$ \\
$\mathrm{N} 10-\mathrm{H} 10 B \cdots \mathrm{O} 22^{\text {viii }}$ & 0.91 & 2.13 & $2.920(6)$ & 145 \\
$\mathrm{~N} 10-\mathrm{H} 10 A \cdots \mathrm{O} 20^{\text {ii }}$ & 0.91 & 2.24 & $2.987(5)$ & 139 \\
$\mathrm{~N} 8-\mathrm{H} 8 B \cdots \mathrm{O} 12^{\mathrm{vi}}$ & 0.91 & 2.04 & $2.937(5)$ & 168 \\
$\mathrm{~N} 8-\mathrm{H} 8 A \cdots \mathrm{O} 23$ & 0.91 & 2.20 & $3.031(5)$ & 152 \\
$\mathrm{~N} 6-\mathrm{H} 6 B \cdots \mathrm{O} 13^{\text {ix }}$ & 0.91 & 2.64 & $3.363(5)$ & 137 \\
$\mathrm{~N} 6-\mathrm{H} 6 B \cdots \mathrm{O} 24^{\text {vi }}$ & 0.91 & 2.24 & $2.984(6)$ & 139 \\
$\mathrm{~N} 6-\mathrm{H} 6 A \cdots \mathrm{O} 13^{\mathrm{v}}$ & 0.91 & 2.25 & $3.158(5)$ & 175 \\
$\mathrm{~N} 4-\mathrm{H} 4 B \cdots \mathrm{O} 2^{\mathrm{x}}$ & 0.91 & 2.33 & $3.182(5)$ & 156 \\
$\mathrm{~N} 4-\mathrm{H} 4 A \cdots \mathrm{O} 27^{\mathrm{v}}$ & 0.91 & 2.18 & $3.037(9)$ & 156 \\
$\mathrm{~N} 4-\mathrm{H} 4 A \cdots \mathrm{O} 25^{\mathrm{x}}$ & 0.91 & 2.01 & $2.789(9)$ & 143 \\
$\mathrm{~N} 2-\mathrm{H} 2 B \cdots \mathrm{O} 27$ & 0.91 & 2.55 & $3.418(9)$ & 159 \\
$\mathrm{~N} 2-\mathrm{H} 2 B \cdots \mathrm{O} 28^{\mathrm{i}}$ & 0.91 & 2.08 & $2.868(9)$ & 144 \\
$\mathrm{~N} 2-\mathrm{H} 2 A \cdots \mathrm{O} 15^{\text {iv }}$ & 0.91 & 2.07 & $2.946(5)$ & 162 \\
\hline
\end{tabular}

Symmetry codes: (i) $-x,-y,-z+1$; (ii) $x, y+1, z$; (iii) $x+1, y+1, z$; (iv) $-x+1,-y+1,-z+1$; (v) $x+1, y, z$; (vi) $-x+1,-y+1,-z$; (vii) $x, y-1, z$; (viii) $x-1, y, z$; (ix) $-x+1,-y,-z$; (x) $-x+1,-y,-z+1$. 